\title{
Genome-wide identification and analysis of WRKY gene family in maize provide insights into regulatory network in response to abiotic stresses
}

Wenjing Hu, Qiaoyu Ren, Yali Chen, Guoliang Xu and Yexiong Qian*

\begin{abstract}
Background: The WRKY transcription factor family plays significant roles in biotic and abiotic stress responses, which has been associated with various biological processes in higher plants. However, very little is known regarding the structure and function of WRKY genes in maize.

Results: In this study, a total of 140 ZmWRKY proteins encoded by 125 ZmWRKY genes were eventually identified in maize. On the basis of features of molecular structure and a comparison of phylogenetic relationships of WRKY transcription factor families from Arabidopsis, rice and maize, all $140 \mathrm{ZmWRKY}$ proteins in maize were divided into three main groups (Groups I, II and III) and the Group II was further classified into five subgroups. The characteristics of exon-intron structure of these putative ZmWRKY genes and conserved protein motifs of their encoded ZmWRKY proteins were also presented respectively, which was in accordance with the group classification results. Promoter analysis suggested that ZmWRKY genes shared many abiotic stress-related elements and hormone-related elements. Gene duplication analysis revealed that the segmental duplication and purifying selection might play a significant role during the evolution of the WRKY gene family in maize. Using RNA-seq data, transcriptome analysis indicated that most of ZmWRKY genes displayed differential expression patterns at different developmental stages of maize. Further, by quantitative real-time PCR analysis, twenty-one ZmWRKY genes were confirmed to respond to two different abiotic stress treatments, suggesting their potential roles in various abiotic stress responses. In addition, RNA-seq dataset was used to conduct weighted gene co-expression network analysis (WGCNA) in order to recognize gene subsets possessing similar expression patterns and highly correlated with each other within different metabolic networks. Further, subcellular localization prediction, functional annotation and interaction analysis of ZmWRKY proteins were also performed to predict their interactions and associations involved in potential regulatory network.
\end{abstract}

Conclusions: Taken together, the present study will serve to present an important theoretical basis for further exploring function and regulatory mechanism of ZmWRKY genes in the growth, development, and adaptation to abiotic stresses in maize.

Keywords: WRKY transcription factor, Expression patterns, Gene duplication, Abiotic stress, Regulatory network analysis, Zea mays $L$

\footnotetext{
* Correspondence: qyx2011@ahnu.edu.cn

Anhui Provincial Key Lab. of the Conservation and Exploitation of Biological

Resources, Anhui Normal University, Wuhu 241000, China
}

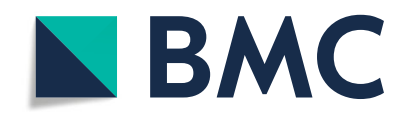

(c) The Author(s). 2021 Open Access This article is licensed under a Creative Commons Attribution 4.0 International License, which permits use, sharing, adaptation, distribution and reproduction in any medium or format, as long as you give appropriate credit to the original author(s) and the source, provide a link to the Creative Commons licence, and indicate if changes were made. The images or other third party material in this article are included in the article's Creative Commons licence, unless indicated otherwise in a credit line to the material. If material is not included in the article's Creative Commons licence and your intended use is not permitted by statutory regulation or exceeds the permitted use, you will need to obtain permission directly from the copyright holder. To view a copy of this licence, visit http://creativecommons.org/licenses/by/4.0/ The Creative Commons Public Domain Dedication waiver (http://creativecommons.org/publicdomain/zero/1.0/) applies to the data made available in this article, unless otherwise stated in a credit line to the data. 


\section{Background}

Maize is the main source of food security and economic development in sub-Saharan Africa and Latin America, and is among the top three crops in Asia [1]. As a result of the global environmental vagaries, various environmental stresses including biotic and abiotic stresses have brought huge threat to the global maize production $[2,3]$. Currently, abiotic stresses, such as extreme temperature, high salinity and drought and so on, have been confirmed to be one of the main factors for the losses of corn yield worldwide. For example, it has been estimated that a one-degree temperature rise reduces maize yields around the world by $7.4 \%$ [4]. Therefore, it is emergent to clarify the molecular mechanism of maize in response to abiotic stresses.

The WRKY TF family is one of the largest transcription factor (TF) families in higher plants [5]. A number of studies have demonstrated that the WRKY transcription factors play critical roles in response to biotic and abiotic stress [6]. The WRKY proteins can induce or repress the expression of their downstream genes by specifically binding to W-box [TGACC $(\mathrm{A} / \mathrm{T})]$ at their promoter sites and eventually activate their stress responses [7]. One of the distinguishing features of the WRKY TFs is the presence of highly conserved WRKY domain. The conserved WRKY domain is composed of approximately 60 amino acid residues with a highly conserved heptapeptides, WRKYGQK, at the Nterminus, and a novel zinc finger motif $\mathrm{C}_{2} \mathrm{H}_{2}\left(\mathrm{C}-\mathrm{X}_{4-5}-\mathrm{C}-\right.$ $\left.\mathrm{X}_{22-23}-\mathrm{H}-\mathrm{X}-\mathrm{H}\right)$ or $\mathrm{C}_{2} \mathrm{HC}\left(\mathrm{C}-\mathrm{X}_{7}-\mathrm{C}-\mathrm{X}_{23}-\mathrm{H}-\mathrm{X}-\mathrm{C}\right)$ at their C-terminus [8-10]. On the basis of the characteristics of WRKY domain and zinc-finger-like motif, the WRKY TFs can be grouped into three major Groups (I, II, and III). The Group II WRKY members are further divided into five subgroups (IIa-IIe) according to their evolutionary divergence $[8,11]$. The Group I members contain two WRKY domains with $\mathrm{C}_{2} \mathrm{H}_{2}$ zinc-finger-like motifs. The Group II members contain a single WRKY domain including a $\mathrm{C}_{2} \mathrm{H}_{2}$ zinc-finger-like motif. The WRKY TFs containing a single WRKY domain with a $\mathrm{C}_{2} \mathrm{HC}$ zinc-finger-like motif belong to the Group III [8].

It has been reported in a great deal of studies that the WRKY genes respond to specific abiotic stresses, such as drought, waterlogging, wounding, and salt stress [12-14]. For example, AtWRKY30 can be induced under various abiotic stresses, meanwhile, the overexpression of AtWRKY3O greatly enhance the resistance of Arabidopsis in response to salt stress [15]. Overexpression of OsWRKY47 can raise the rice production, and the tolerance of rice to drought also can be improved compared with normal rice plants [16]. Additionally, a great amount (54/103) of OsWRKY genes showed remarkable changes in expression levels after salt, drought and cold stresses treatments [17]. Moreover, in wheat, the majority ( 8 out of 15 ) of TaWRKY genes were transcribed in response to cold, heat, salt and PEG treatments [18].
As far as we know, only few WRKY genes have been reported in maize. ZmWRKY17 could control the transcription of some stress- and ABA-related genes, and eventually improved the salt stress resistance and reduced ABA sensitivity [19]. ZmWRKY33 could be activated by some abiotic stress such as high-salt, dehydration, cold, and ABA treatments, and it increased the salt stress resistance of transgenic Arabidopsis [20]. In addition, ZmWRKY58 enhanced both salt and drought stress tolerance of transgenic rice [21]. These studies demonstrated that the role of $Z m W R K Y \mathrm{~s}$ in terms of enhancing tolerance to abiotic stresses. Although over 100 members of the maize WRKY gene family have been proposed [22], the expression patterns of $Z m W R K Y \mathrm{~s}$ in different tissues of maize under abiotic stresses have not been investigated at genome-wide level. Many details about maize WRKY gene family remain to be further elucidated.

In this study, 140 WRKY TF proteins were identified from the latest B73 maize genome database and orderly named. Moreover, a comprehensive analysis of $Z m W R K Y$ genes was accomplished including their phylogenetic relationship, chromosome location, gene duplication, exonintron structure, cis-acting factors, conserved domains and expression patterns in various tissues and under salt and drought stress treatments. Furthermore, weighted gene co-expression network analysis was used to identify modules of co-expression network and explore key genes involved in plant development. Further, subcellular localization and Gene ontology (GO) annotation were performed using the online WOLF PSORT software and Blast2GO software to analyze functional classification of ZmWRKY proteins in maize respectively and PPI (protein-protein interaction) network was constructed using the Search Tool for the Retrieval of Interacting Gene (STRING) database to further understand the biological and molecular functions of ZmWRKY proteins. Taken together, these results may provide an in-depth understanding of the evolution of WRKY gene family in maize and their critical roles played in abiotic stress responses.

\section{Results}

\section{Identification and analysis of WRKY genes in maize}

In this study, a set of 140 WRKY TF proteins were identified through the maize genomic database (Table S1). A total of $125 W R K Y$ genes were consistently named as ZmWRKY1- ZmWRKY125 based on their chromosome location, while the variant proteins generated from the same locus were granted by the same name followed by 1,2 or 3 . The characteristics were analyzed including the genome location of these identified genes, length of the open reading frame (ORF), basic information of their encoded proteins including length, molecular weight (MW) and isoelectric point (pI). As shown in the Table S1, the 
length of ZmWRKY proteins varied from 99 (ZmWRKY7) to 729 (ZmWRKY59) amino acids and the average protein sequence length is 349 residues. The pI ranged from 4.5514 (ZmWRKY98) to 10.7787 (ZmWRKY125), and the MW (molecular mass) ranged from 11,218.7 Da (ZmWRKY7) to 78,734.7 Da (ZmWRKY59).

\section{Classification of maize WRKY proteins}

To analyze the phylogenetic relatedness among ZmWRKY proteins in maize, a total of 156 conserved WRKY domains including two WRKY domains of the Group I members, were extracted to construct the evolutionary tree using the neighboring method. To obtain a more precise result, 16 OsWRKY proteins from rice (O. sativa japonica) and 15 AtWRKY proteins from Arabidopsis were analyzed together with all ZmWRKY proteins identified in this study. The WRKY domain sequences of candidate rice and Arabidopsis WRKY proteins were downloaded from the Smart database (http://smart.emblheidelberg.de/). As depicted in the constructed evolutionary tree (Fig. 1), the 140 ZmWRKY proteins were classified into three main Groups (I, II and III), and the Group II proteins were further categorized into 5 subgroups (IIa, IIb, IIc, IId and IIe). To reveal how conservative the heptapeptide WRKYGQK and zinc-finger-like domains were in each group, the sequence logos were produced by the WebLogo online program to exhibit the conservation at each residue position, and the sequence alignment were further performed by DNAMAN 7.0 (Fig. S1). The WRKY TFs have two standard motifs, and the first one WRKYGQK sequence can combine with the $\mathrm{W}$ box cis-element to induce their downstream gene expression. Besides the WRKYGQK sequence, three variants, WKKYGQK (ZmWRKY7 and - 62) and WRKYGKK (ZmWRKY26, - 41, - 71.1, - 71.2, - 76, - 89, - 94 and$100)$ in the Group IIc and WRKYGEK (ZmWRKY1, - 19, $-55,-57,-97$ and -117$)$ in the Group III, were also revealed in the ZmWRKY gene family. The other one was a zinc-finger-like domain with two types, namely $\mathrm{C}_{2} \mathrm{H}_{2}$ and $\mathrm{C}_{2} \mathrm{HC}$.

As shown in Fig. S1, it is worth noting that the Group I was clustered by 25 WRKY proteins, among which there were sixteen members containing two WRKY domains. Fifteen of these proteins contained two intact WRKY domains, however ZmWRKY116.1 only contained a single

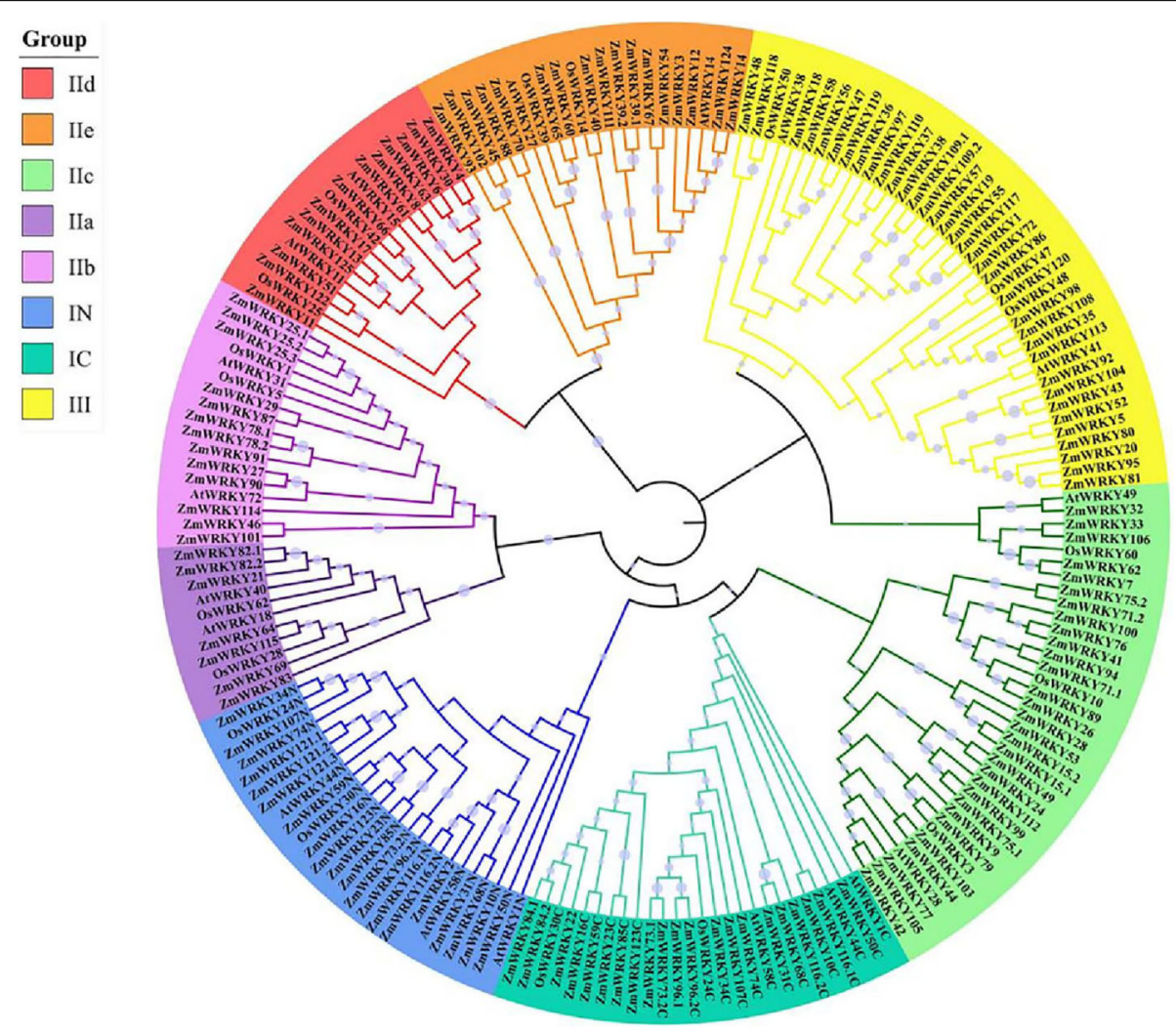

Fig. 1 Phylogenetic analysis among WRKY domains of WRKY proteins of maize, rice and Arabidopsis. The Unrooted neighbor-joining (NJ) phylogenetic tree was constructed with WRKY domains of WRKY proteins from maize, rice and Arabidopsis using MEGA7.0 with a bootstrap of 1000. Gray circles of different sizes represent the level of support. Eight main clades are marked: IN, IC, Ila, IIb, IIc, IId, Ile, and III with different colored ranges. Protein sequences were downloaded from National Center for Biotechnology Information (NCBI) and Maize genome database. The respective WRKY domain sequences of candidate WRKY proteins were downloaded from the Smart database 
intact WRKY domain in its N-terminal, while its Cterminal domain lacked a zinc-finger domain. In addition, the other nine members had only a single WRKY domain at either the C-terminal (ZmWRKY22, - 73.1, - 84.1, 84.2 and -96.1) or the N-terminal (ZmWRKY2, - 121.1, -121.2, -121.3) of these proteins, suggesting that they probably had gone through domain loss or acquisition events during their evolutionary process [23]. Moreover, the zinc-finger motifs of the ZmWRKYs in the Group I belonged to the $\mathrm{C}_{2} \mathrm{H}_{2}$ type with a C- $\mathrm{X}_{4}-\mathrm{C}-\mathrm{X}_{22-23}-\mathrm{H}-\mathrm{X}_{1}-\mathrm{H}$ motif (Fig. S1). There were 80 members assigned to the Group II, while 75 of them contained the motif of C- $\mathrm{X}_{4-5^{-}}$ $\mathrm{C}-\mathrm{X}_{23-24}-\mathrm{H}-\mathrm{X}_{1}-\mathrm{H}$, and three members, ZmWRKY33, -87 and -114, lacked a typical zinc-finger-like motif. All the Group II 80 members were further divided into five subgroups according to their phylogenetic relationship. The subgroups were distributed as the follows: the Group IIa (7 proteins), the Group IIb (13 proteins), the Group IIc (30 proteins), the Group IId (13 proteins), and the Group IIe (17 proteins). The zinc-finger motifs of the Group III members (35) belonged to the $\mathrm{C}_{2} \mathrm{HC}$ type, with the C- $\mathrm{X}_{5-}$ 7-C-X $\mathrm{X}_{23-38}-\mathrm{H}-\mathrm{X}_{1}-\mathrm{C}$ motif (Fig. S1), except ZmWRKY48 and-117 only containing fragmentary zinc-finger structure.

\section{Analyses of chromosomal location, gene duplication and genome synteny}

The genomic distribution of ZmWRKY genes in maize was carried out by MapInspect software. A total of 125 candidate $Z m W R K Y$ genes were mapped to all 10 chromosomes of maize with an uneven distribution (Fig. 2). The chromosome 8 contained the largest number of $Z m W R K Y$ s (26 genes) and the second was the chromosome 3 (24 genes). The least number of ZmWRKYs was found on the chromosome 9, with only five genes. All chromosomes contained the members from all three groups in addition to the chromosome 5, which only had the Group II members.

The gene duplication events were analyzed to reveal the expansion mechanism of the maize WRKY gene family. Among the $Z m W R K Y$ genes, totally 52 gene pairs were involved in gene duplication events. Holub [24] defined the tandem duplication event as a chromosome region within $200 \mathrm{~kb}$ including two or more genes. All the 52 ZmWRKY gene pairs among 78 WRKY genes (Table S4) were recognized as segmental duplication, but no tandem duplication was identified, indicating that tandem duplication events might have not taken part in the amplification of ZmWRKY gene family. Most of the duplications were located between the chromosomes 3 and 8 (Fig. 3).

To further estimate the origin and evolutionary history of the ZmWRKY gene family, two comparative syntenic maps were constructed among maize, rice and Arabidopsis at genome-wide levels, respectively. As shown in Fig. S2, we finally identified 132 orthologous gene pairs between maize and rice, but only 6 orthologous gene pairs between maize and Arabidopsis. More details about these orthologous gene pairs were shown in Table S5 and S6. There were far more orthologous genes between maize and rice than that between maize and Arabidopsis, which was probably resulted from the nearer phylogenetic relationship between maize and rice [23].

To determine the selection pressure on different duplicated $W R K Y$ genes, the $K a$ and $K s$ substitution rates and the $K a / K s$ ratios for each repeat $Z m W R K Y$ gene pair were calculated, respectively. Normally, a ratio of 1 indicates neutral selection; a $\mathrm{Ka} / \mathrm{Ks}$ ratio $>1$ means adaptive evolution with positive selection, while a ratio $<1$ means negative or purifying selection - i.e. evolutionary pressure to conserve the ancestral state [25]. As a result, all the $K a / K s$ ratios for the 52 segmentally duplicated gene pairs were $<1$ (Table S4), indicating that the maize WRKY gene family is highly conserved during evolution. According to a substitution rate of $6.5 \times 10^{-9}$ substitutions per synonymous site per year, the divergence time of duplicated $Z m W R K Y$ gene pairs was estimated to range from 9.6653 to 153.9372 Mya (Table S4).

\section{Gene structure and conserved motifs analysis of ZmWRKY genes}

The diversity of gene structure promotes the evolutionary process of the large gene families. To obtain a much more in-depth insight on evolution of the WRKY gene family in maize, we mapped the genetic structure of each ZmWRKY gene. A phylogenetic tree was constructed according to the full-length ZmWRKY proteins (Fig. 4) to better analyze gene structure and conserved motifs. The number of introns in $Z m W R K Y$ genes ranged from 0 to 5. The majority of $Z m W R K Y$ genes contained one to three introns, for 78 members containing two introns; 26 containing one intron; and 13 containing three introns. The other 8 and 7 $Z m W R K Y$ genes contained four and five introns, respectively. Additionally, the remaining $8 Z m W R K Y$ genes contained no intron. As shown in Fig. 4, most ZmWRKY genes within the same group or subgroups contained a similar gene organization, indicating the functional similarity shared among different members.

To gain a better understanding of the similarity and dissimilarity of motifs in different ZmWRKY proteins, 20 conserved motifs were identified within proteins by the MEME program. MEME motif analysis exhibited that each ZmWRKY protein had its specific conserved motifs (Table S2). As depicted in Fig. 4, the WRKY family members with similar motif structures were divided into the same group. Almost all ZmWRKYs had the conserved heptapeptides WRKYGQK (Motif 1 or Motif 3 ), and all contained at least one motif. Moreover, the 

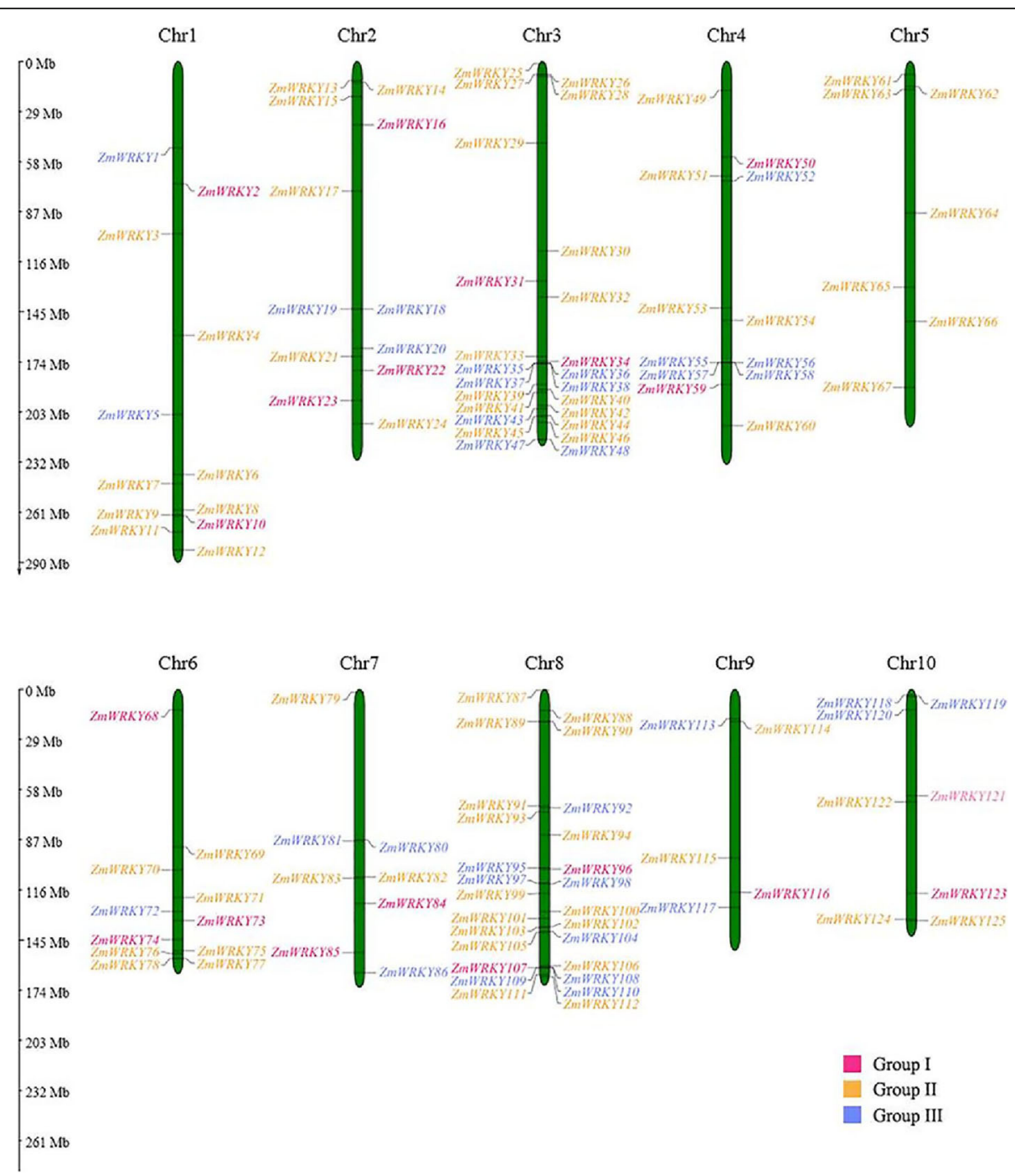

Fig. 2 Chromosomal location of WRKY family genes in maize. Distribution of WRKY genes in maize chromosomes. A total of $125 \mathrm{ZmWRKY}$ genes were mapped on the ten maize chromosomes with an uneven distribution. The chromosome numbers are indicated at the top of each vertical gray bar. The gene names on the both side of each chromosome correspond to the approximate locations of each ZmWRKY genes. The scale on the left is in megabases

Motif 2 was comprised of the $\mathrm{C}_{2} \mathrm{H}_{2}$ motif, while the Motif 11 was made up of the $\mathrm{C}_{2} \mathrm{HC}$ motif. The conserved motif analysis demonstrated that the conserved motifs were specifically present in different groups. For instance, the Group IIa members contained 4 conserved motifs (Motifs 1, 2, 8 and 12); 13 members of the Group IIb contained 7 conserved motifs (Motifs 1, 2, 4, 8, 10, 12 and 15); the members of the Group IId contained 5 conserved motifs (Motifs 1, 2, 6, 14 and 17). It was clear that a few motifs particularly existed in one or more groups and subgroups. For instance, the Motifs 3, 5 and 16 existed in the Group I members, and the Motifs 9 and 11 existed only in the Group III, while the Motif 17 was mainly present in the Group IId. This result indicated the different roles that these groups might play in evolution and function.
The cis-elements in the promoters of maize WRKY genes In order to confirm the potential function of $Z m W R K Y$ genes in abiotic stress responses, the $2000 \mathrm{bp}$ promoter sequences of the $Z m W R K Y$ genes were extracted and analyzed for cis-elements using the PlantCARE database. As depicted in Fig. S3, totally eleven types cis-acting elements in relation to stresses and phytohormone responses were discovered in the promoters of $Z m W R K Y$ genes, including four abiotic stress-related elements (W box, MBS, LTR and TC-rich repeats) and seven hormone-related elements (ERE, ABRE, GARE-motif, TCA-element, TGA-element, CGTCA-motif and TGACG-motif). As shown in Fig. S4, the CGTCA-motif and TGACG-motif (MeJA-responsive elements) were the most numerous elements in the pro-

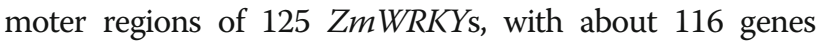
containing these two elements. ABRE (ABA-responsive 


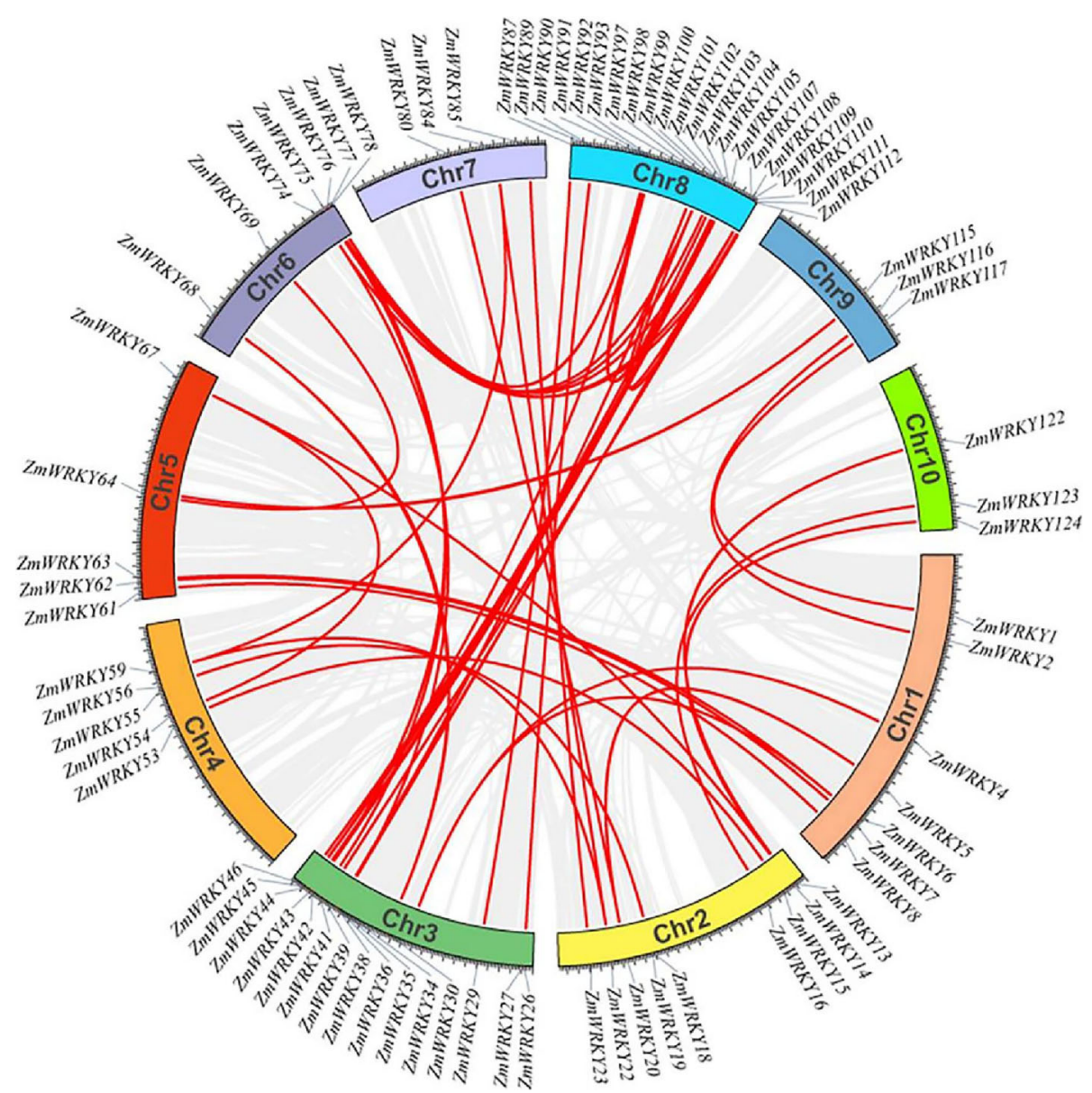

Fig. 3 Synteny analysis of ZmWRKY genes in maize genome. Duplicated blocks in maize chromosomes were revealed. The circular image retrieved from PLAZA database show inter-chromosome homologous regions connected by bands in different colors. The chromosome numbers and ZmWRKY genes are indicated outside. Gray lines mean all syntenic regions in whole maize genome and red lines mean ZmWRKY gene pairs with segmental duplication

elements) also appeared frequently in $\operatorname{ZmWRKY}$ gene promoter regions, which was found in 114 promoters. Several W-boxes were detected in $88 \mathrm{ZmWRKY}$ gene promoters, suggesting that these genes have the likelihood to be regulated by other WRKY TFs or themselves. LTR and MBS elements that respond to low temperature and drought stresses were detected in the promoters of 56 and 63 ZmWRKY genes, respectively. Thirty promoters had a GARE-motif (gibberellin- responsive element) and only 19 promoters showed a TC-rich repeats element (cis-acting element participated in defense and stress response). Therefore, the cis-element analysis revealed that the expression of $Z m W R K Y$ genes in maize might be associated with different environmental factors.

\section{Analysis of expression profiles of maize WRKY genes in different tissues}

In this study, to investigate the potential functions of putative ZmWRKY genes in plant growth and development, the expression profiles of $Z m W R K Y s$ in 10 tissues at different developmental stages were analyzed according to the microarray data (Fig. 5). However, the expression data for only $135 \mathrm{ZmWRKY}$ transcripts was revealed as shown in Table S7. The expression data of other five transcripts (ZmWRKY25.1, - 25.2, - 25.3, - 32 and - 106) were not detected, indicating that these genes may be pseudogenes or have particular temporal and spatial expression patterns not explored in this database. All $135 \mathrm{ZmWRKY}$ transcripts investigated were expressed in all tissues, although most members were only expressed at low levels, which suggested that these TFs might work with other proteins in a synergistic or interactive manner during plant growth and development. Notably, $15 \mathrm{ZmWRKY}$ transcripts $(Z m W R K Y 2,-22,-23,-31,-50,-68,-85$, -116.1 and -116.2 from the Group I, ZmWRKY6, - 8, 30, -61 and -63 from the Group IId, and ZmWRKY100 from the Group IIc) exhibited high expression levels in all ten tissues, suggesting that these genes might play a fundamental role in the maize growth and development. What's more, a few genes exhibited preferential 


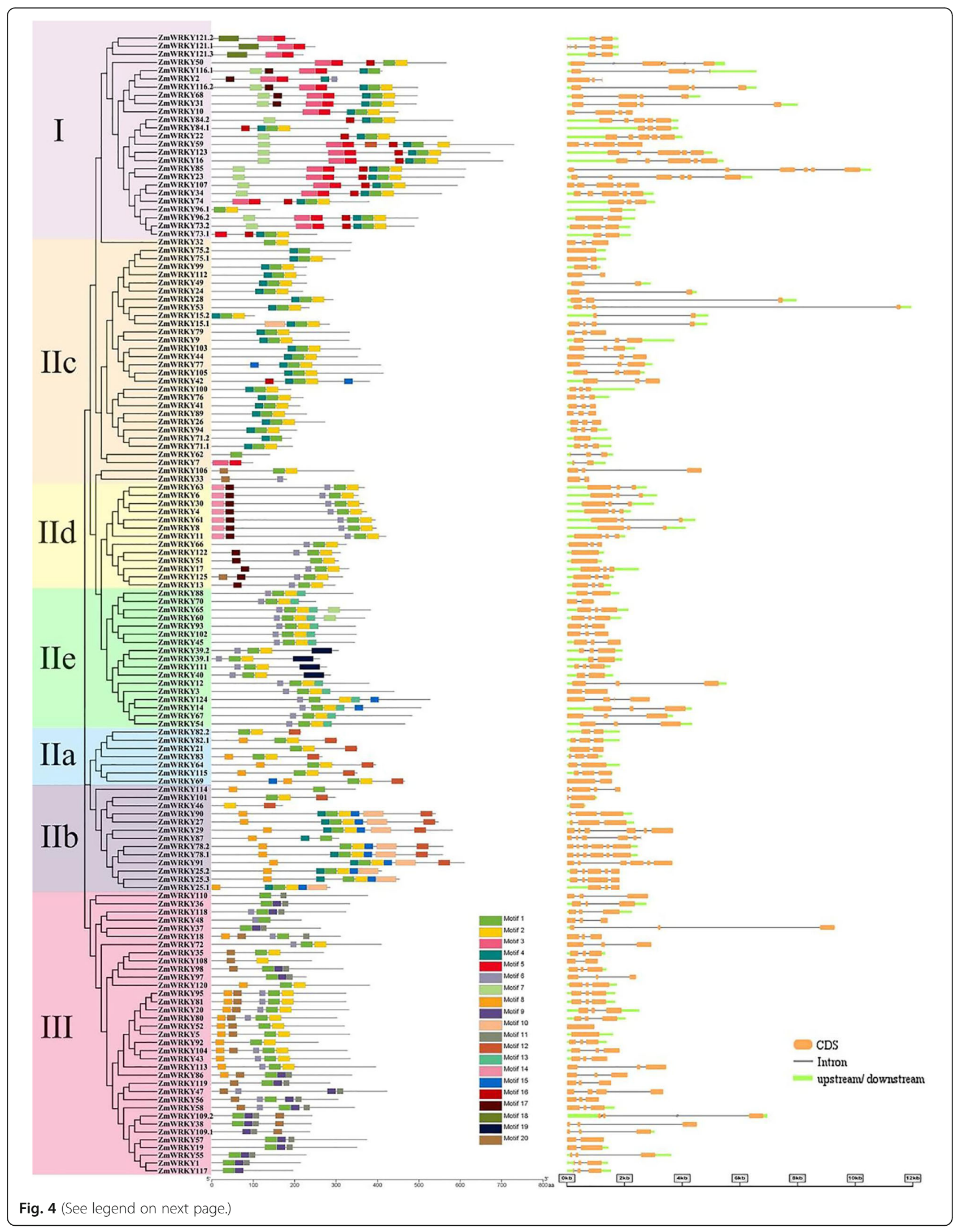


(See figure on previous page.)

Fig. 4 Unrooted phylogenetic tree, gene structures and conserved motifs of the ZmWRKY family in maize. The evolutionary tree from the ZmWRKY protein sequences was constructed using the MEGA 7.0 program with the Neighbor-joining method. Different colors represented various groups. The conserved motifs of ZmWRKY proteins were analyzed using MEME. Boxes with different colors denote different motifs and their position in each ZmWRKY protein. All 125 genes' structures were obtained using GSDS 2.0. CDS, intron and 5'/3' UTR are denoted by orange rectangles, single lines and green rectangles, respectively. (Color figure online)

expression in various tissues. For example, $Z m W R K Y 30$, ZmWRKY55, ZmWRKY59 and ZmWRKY118 showed the highest transcript abundances in husk, silks, seed and stem, respectively. In addition, several genes were discovered to be highly expressed in roots particularly. These genes might share special functions in the specific tissues. Additionally, several gene pairs with close relationship, such as ZmWRKY23/85, ZmWRKY31/68, ZmWRKY81/95 and $Z m W R K Y 16 / 123$, exhibited similar expression profiles, indicating that the functionality of these genes might be redundant.

\section{Expression profiles of maize WRKY genes under abiotic stress treatment}

Under unfavorable circumstance, many stress-induced genes are in respond to help plants defend various adversity stresses. To further certify the stressresponsiveness of ZmWRKY genes to abiotic stresses, quantitative RT-PCR was accomplished for twenty-one $Z m W R K Y$ genes randomly chosen from the Group III and the Group IId, including 8 Group IId members and 13 Group III members (Figs. 6 \& 7). The seedling leaves and roots were sampled at different time points to analyze their dynamic response to salt and drought stresses using qRT-PCR (Figs. 6 \& 7). Under salt treatment, ten $Z m W R K Y$ transcripts $(Z m W R K Y 5,-17,-35,-51,-63$, $-80,-92,-108,-119$ and -122) didn't show upregulation in leaves, but they were all up-regulated in roots at different time points, while only one $Z m W R K Y$ transcript (ZmWRKY86) exhibited down-regulation in roots at different time points and most of the remaining $Z m W R K Y$ genes showed significant up-regulation in roots

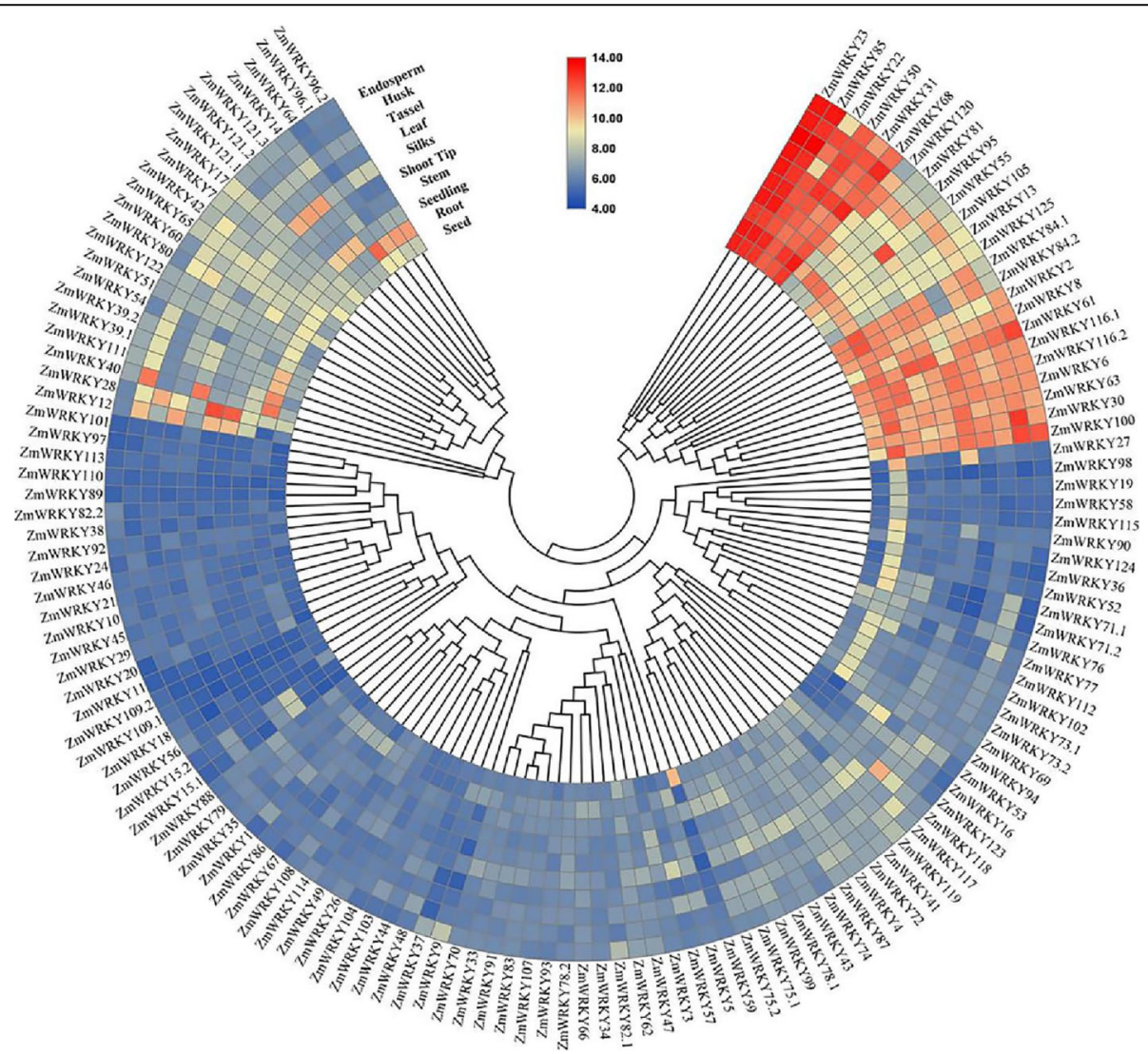

Fig. 5 Hierarchical clustering of expression profiles of ZmWRKY gene family in 10 tissues. Ten tissues from different developmental stages including endosperm, husk, tassel, leaf, silks, shoot tip, stem, seedling, root and seed were investigated. The expression values were shown as $\log 2$ of the RPKM values. The scale bar is shown in the middle and higher expression levels are presented as red color 


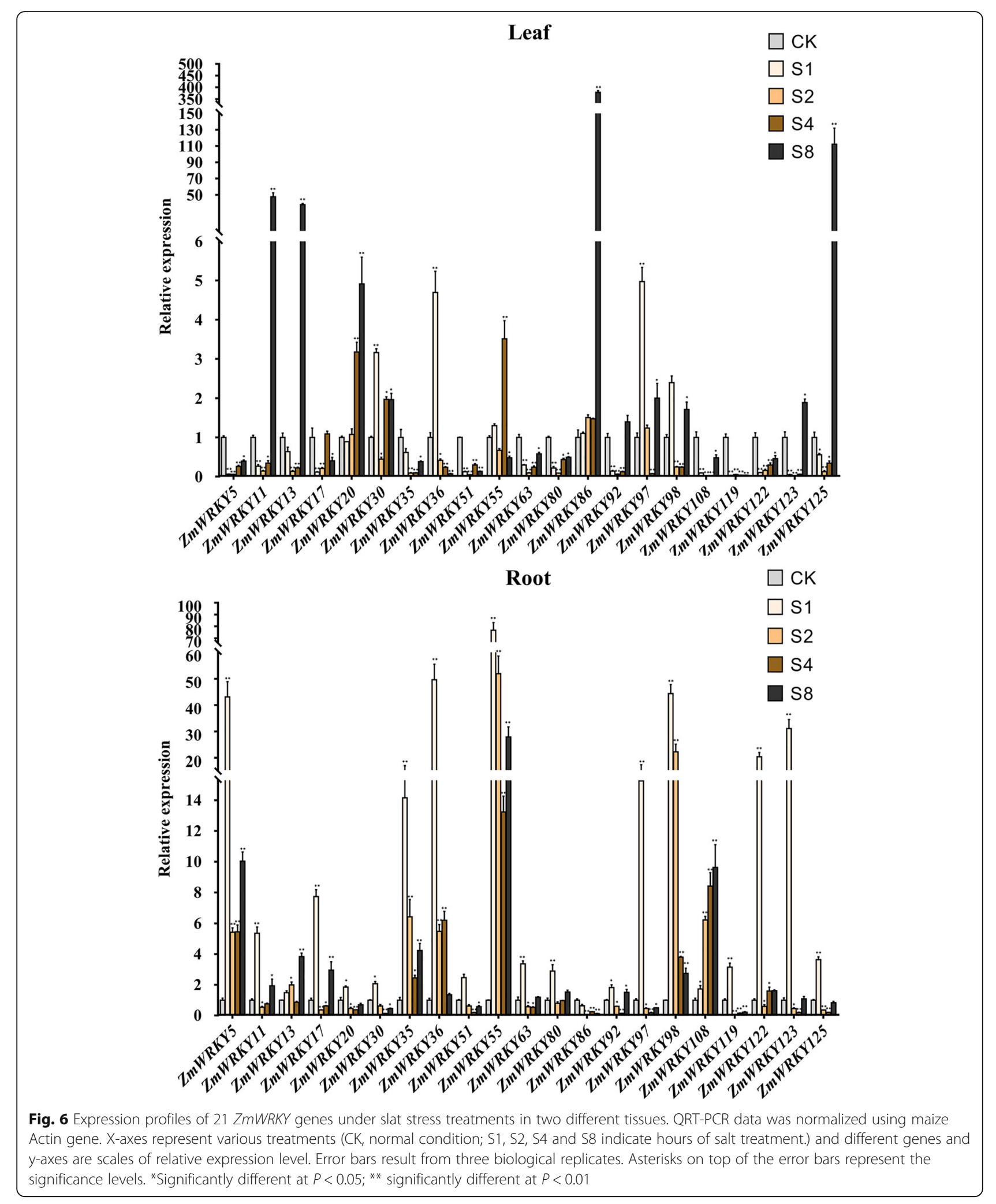




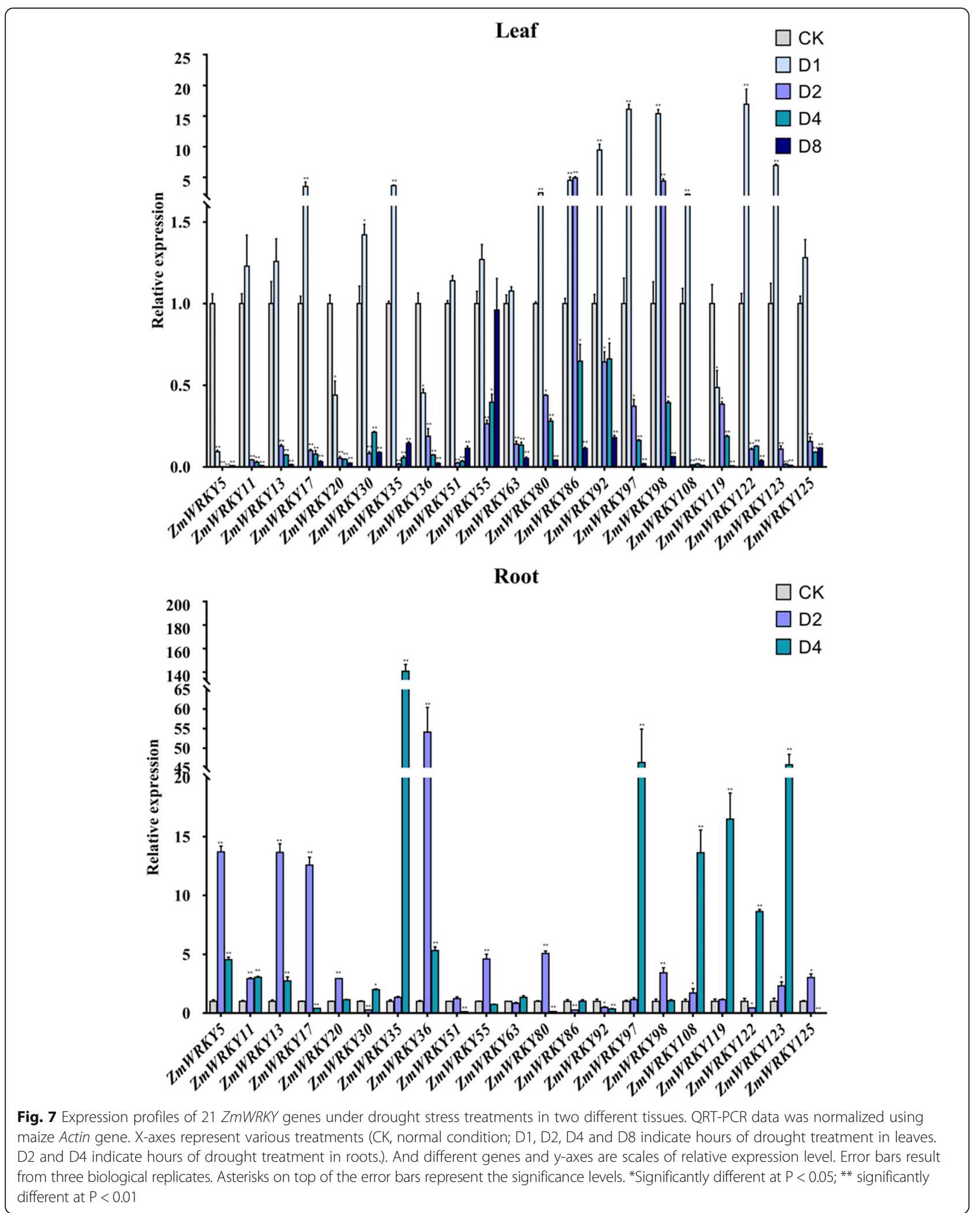


after salt treatment, indicating that these genes may play important roles in the response to salt stress. Furthermore, under salt treatment, most of these genes exhibited more rapid and much stronger responses in roots than that in leaves. For example, there were only $4 \mathrm{ZmWRKY}$ genes $(Z m W R K Y 11,-13,-86$ and -125$)$ responding to salinity and peaked at 10 -fold greater levels at $8 \mathrm{~h}$ after salt treatment in leaves, but $8 \mathrm{ZmWRKY}$ genes were shown to exhibit a high expression level and rapidly peaked at 14fold greater levels at $1 \mathrm{~h}$ after salt treatment in roots. Among them, the transcription level of ZmWRKY55 was up-regulated and peaked at 80 -fold greater levels at $1 \mathrm{~h}$. Furthermore, we further examined the responsiveness of these selected genes in response to drought stress, and obtained a similar expression pattern either under drought treatment or under salt treatment. Likewise, under drought treatment, in leaves, most genes exhibited an upregulation in varying degrees after $1 \mathrm{~h}$ of drought treatment but were down-regulated with the prolonging of treatment time. Interestingly, no matter under drought treatment or salt treatment, the responses of these genes in roots were much stronger than that in leaves. For example, there were only $4 \mathrm{ZmWRKY}$ genes (ZmWRKY92, - 97, - 98 and -122) exhibiting a high expression level (increased $>10$ folds) in leaves under drought treatment, but $9 \operatorname{ZmWRKY}$ genes were expressed highly at 10-fold greater levels in roots. The transcript level of ZmWRKY35 even changed at 150 -fold greater levels in roots. These results suggested that $Z m W R K Y$ genes in roots might participate in plant responses to salt stress and dehydration more strongly or rapidly than that in leaves when respond to these stress conditions.

\section{Weighted gene co-expression network analysis}

To further confirm gene association patterns between different samples and identify highly coordinated genes, a matrix with 60 samples in row names 135 genes in column names was obtained from the database to perform a co-expression network analysis using Weighted Gene Co-Expression Network Analysis. The standardized sample gene expression profile matrix was used as the input file for co-expression network construction. By constructing a hierarchical clustering tree of 135 genes from 60 tissue samples in maize, it was revealed that there were no obvious outliers (Fig. S5). In order to be more accord with the scale-free features, a $\beta$ value of 5 was selected to construct a co-expression network. Through the dynamic cutting tree method for module identification, a total of 7 modules were obtained (Fig. S6). The genes that could be included in any module were placed in the gray module and deleted in the subsequent analysis. The TOM heatmap was designed according to the interaction relations among the 7 modules (Fig. S7). The results showed that each module has been independently authenticated with another module, suggesting the high degree of individuality between the modules and the relative independence of gene expression in each module. In addition, the eigengenes were also calculated and clustered according to their correlation to explore the co-expression similarity of modules. We found that these 7 modules were mainly divided into three clusters (Fig. 8). The heat map drawn based on the adjacency relationship exhibited similar results.

In Fig. 9, it was revealed that there were 10 genes identified in black module specific to the leaf (V9) across all the developmental stages. Of them, ZmWRKY120 was highly expressed in most of tissues. In the network of black module, ZmWRKY21, ZmWRKY82.1, $Z m W R K Y 82.2$ were highly related to ZmWRKY83. The blue module (27 genes) was associated with the leaves (VT and R1). The brown module, representing 16 genes, was highly associated with the stems (V1, V3 and V4). The network of brown module showed that ZmWRKY53 was highly related to ZmWRKY15.1 and ZmWRKY15.2. The green module, containing 15 genes, was related to the husks (R1 and R2) and periearp. In particular, three genes (ZmWRKY55, ZmWRKY100 and ZmWRKY105) showed high expression levels in all tissues. The red module (15 genes) and turquoise module (31 genes) were highly associated with the root (V1), leaves (V3, V9, VT and R2) and husk (R2). In the network of red module, $Z m W R K Y 22$ was highly related to $Z m W R K Y 84.1$ and ZmWRKY84.2. The last module, yellow module (12 genes), was related to the roots (V1 and VE). Among them, ZmWRKY51 and ZmWRKY112 showed high expression levels in all tissues.

\section{Subcellular localization of ZmWRKY proteins}

In view of the fact that subcellular location information can provide some clues for protein function research, the online software Wolf PSORT was used to predict subcellular locations of ZmWRKY proteins in this study (Table S8). The prediction results of subcellular localization indicated that most ZmWRKY proteins are mainly located in the nucleus, while there are still a few ZmWRKY proteins located in various organelles such as chloroplast, mitochondrion, cytoplasm, endoplasmic reticulum and extracellular. The results revealed that these newly-identified ZmWRKY proteins in maize exhibited a various subcellular distribution, which may be associated with functional diversification in abiotic stress responses.

\section{GO annotation and interaction analysis of specific ZmWRKY proteins}

To explore the biological and molecular functions of ZmWRKY proteins, GO annotation and PPI analysis 


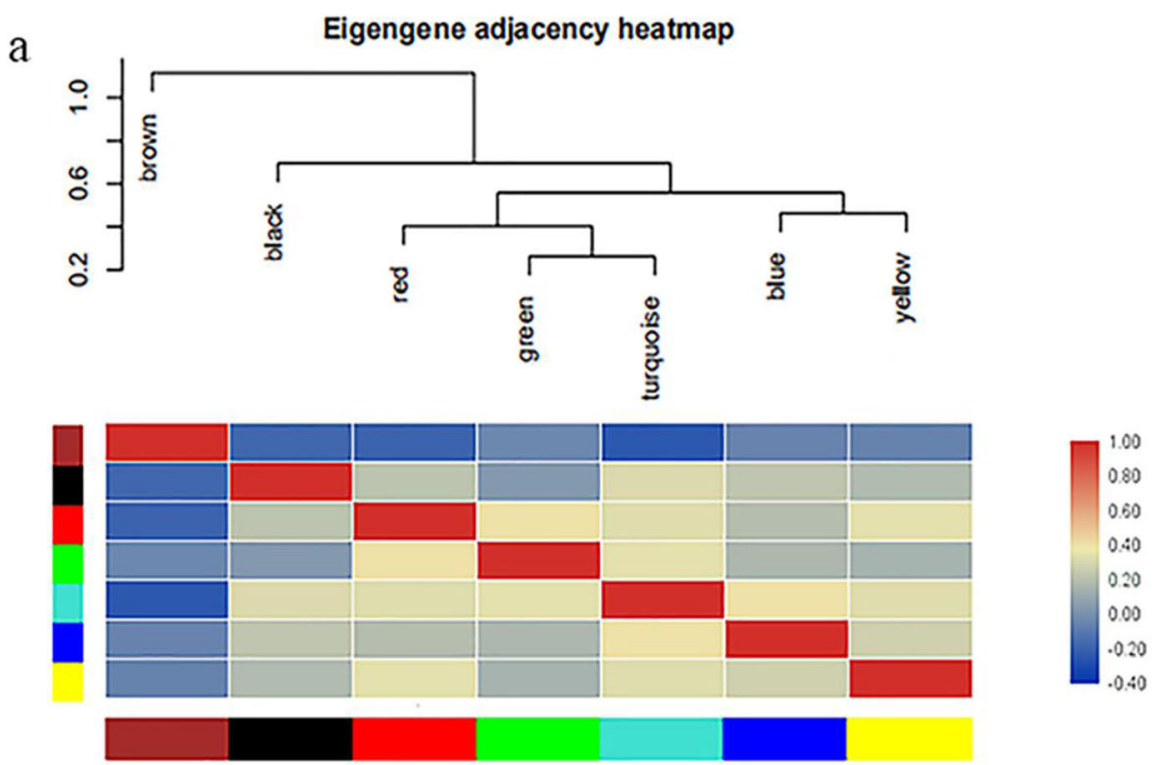

$\mathrm{b}$

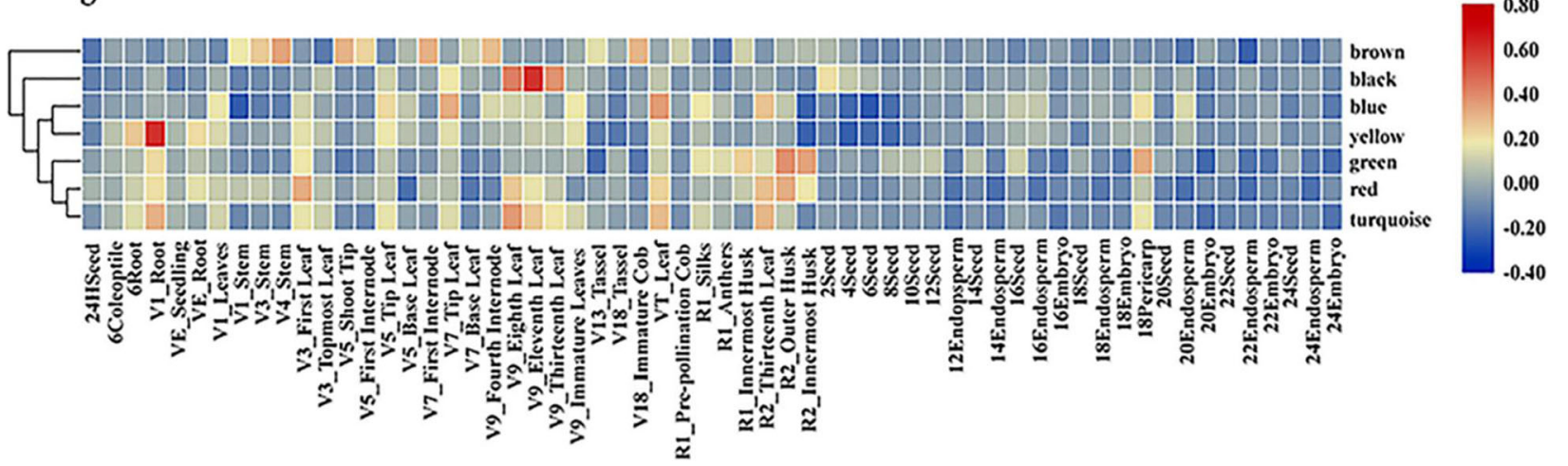

Fig. 8 Co-expression network analysis of ZmWRKY genes. (a) Elgengene adjacency heatmap. A high degree of correlation between modules is indicated by red. (b) Module sample correlation. Each row corresponds to a module. Each column corresponds to specific tissue. The color of each cell at row-column intersection indicates the correlation coefficient between the module and the tissue. A high degree of correlation between a specific module and tissue type is indicated by red

were further conducted in this study. The GO enrichment analysis was composed of three parts: biological process $(\mathrm{BP})$, cellular component $(\mathrm{CC})$, and molecular function (MF). Among the protein sequences annotated by the GO database, a total of 121 ZmWRKY proteins were divided into 3 categories and 20 subcategories (Fig. 10). In the biological process category, the proteins were distributed into 10 subcategories, and the major subcategories were 'metabolic process' (GO: 0008152, 121 sequences, 100\%), 'cellular process' (GO: 0009987, 121 sequences, 100\%) and 'regulation of biological process' (GO: 0050789, 120 sequences, 99.2\%), followed by 'biological regulation' (GO: 0065007, 120 sequences, 99.2\%). In the Cellular Component, 'cell' (GO: 0005623), 'organelle' (GO: 0043226) and 'cell part' (GO: 0044464), with each having 120 (99.2\%) sequences. In the molecular function category, 'organic cyclic compound binding' (GO: 0005488, 121 sequences, 100\%) and 'nucleic acid binding transcription factor activity' (GO: 0001071, 120 sequences, 99.2\%) had the highest representation.

Moreover, a protein-protein interaction network of 17 ZmWRKY proteins involved in stress responses was constructed using STRING 10.5 software based on the Arabidopsis association model (Fig. 11). Among the 17 ZmWRKY proteins, there were five proteins belonging to the Group I, eight proteins belonging to the Group II, and two proteins belonging to the Group III, respectively. In addition, AtWRKY40 (ZmWRKY21, ZmWRKY81.1 and ZmWRKY81.2), AtWRKY33 (ZmWRKY34 and ZmWRKY107), AtWRKY30 (ZmWRKY113), AtWRKY18 (ZmWRKY21, ZmWRKY81.1 and ZmWRKY81.2) and AtWRKY70 (ZmWRKY120) were also involved in a stronger interaction network with other proteins. 

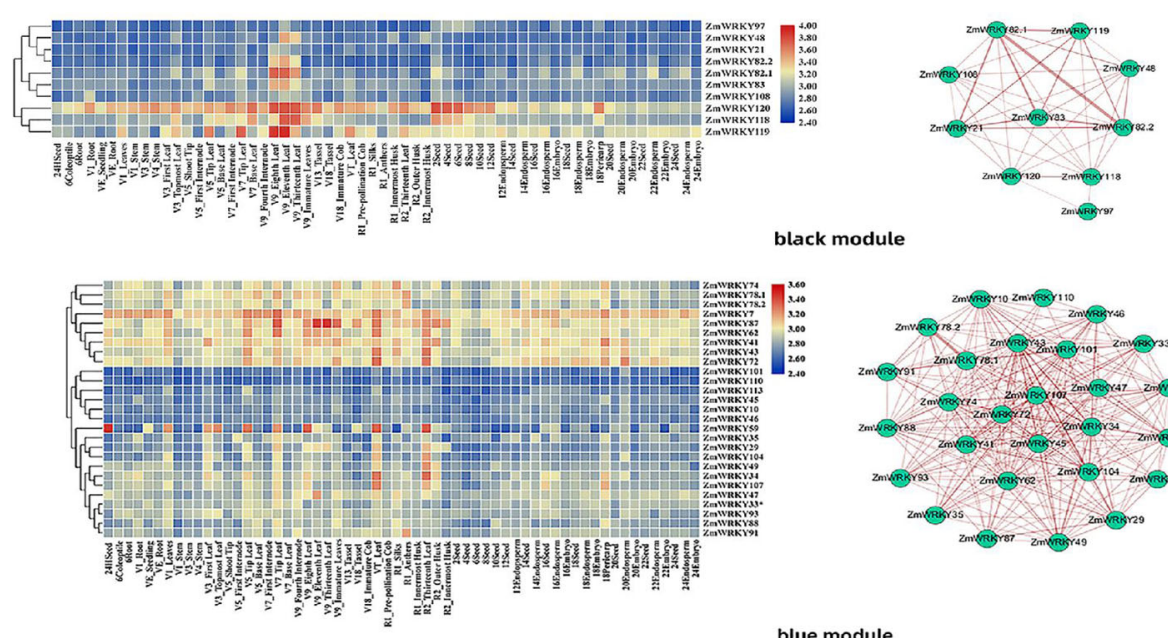

black module

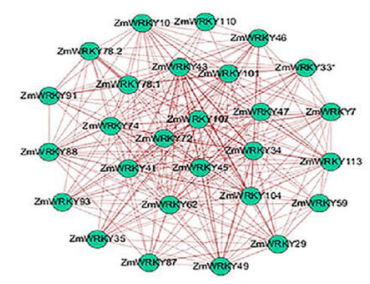

blue module
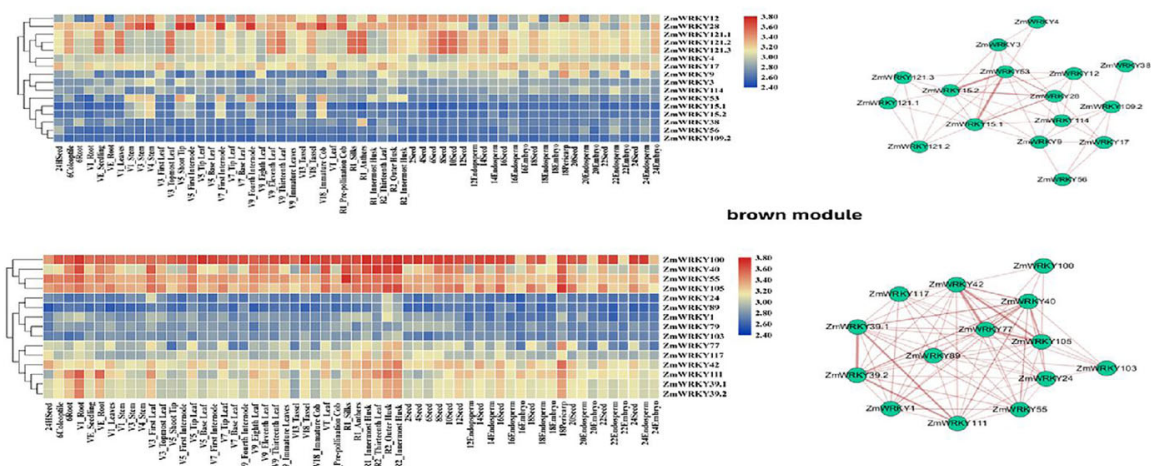

green module

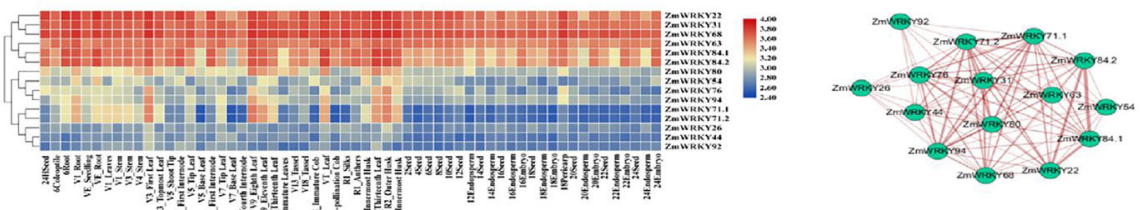

red module
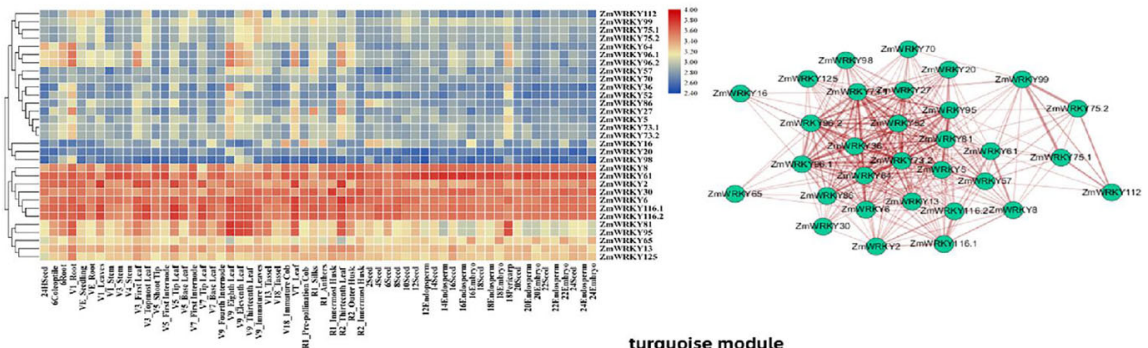

turquoise module

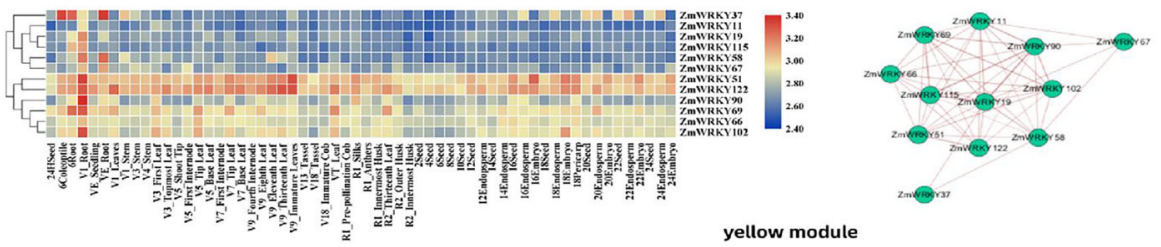

Fig. 9 Co-expression network analysis of modules of ZmWRKY genes. Heatmaps showing genes in each module that were expressed in tissues. Right correlation networks in the module corresponding to heatmap. Network is visualized in Cytoscape. Green and red color Spheres (nodes) represent $Z m W R K Y$ genes, and the thick lines (edges) represent the high correlation 

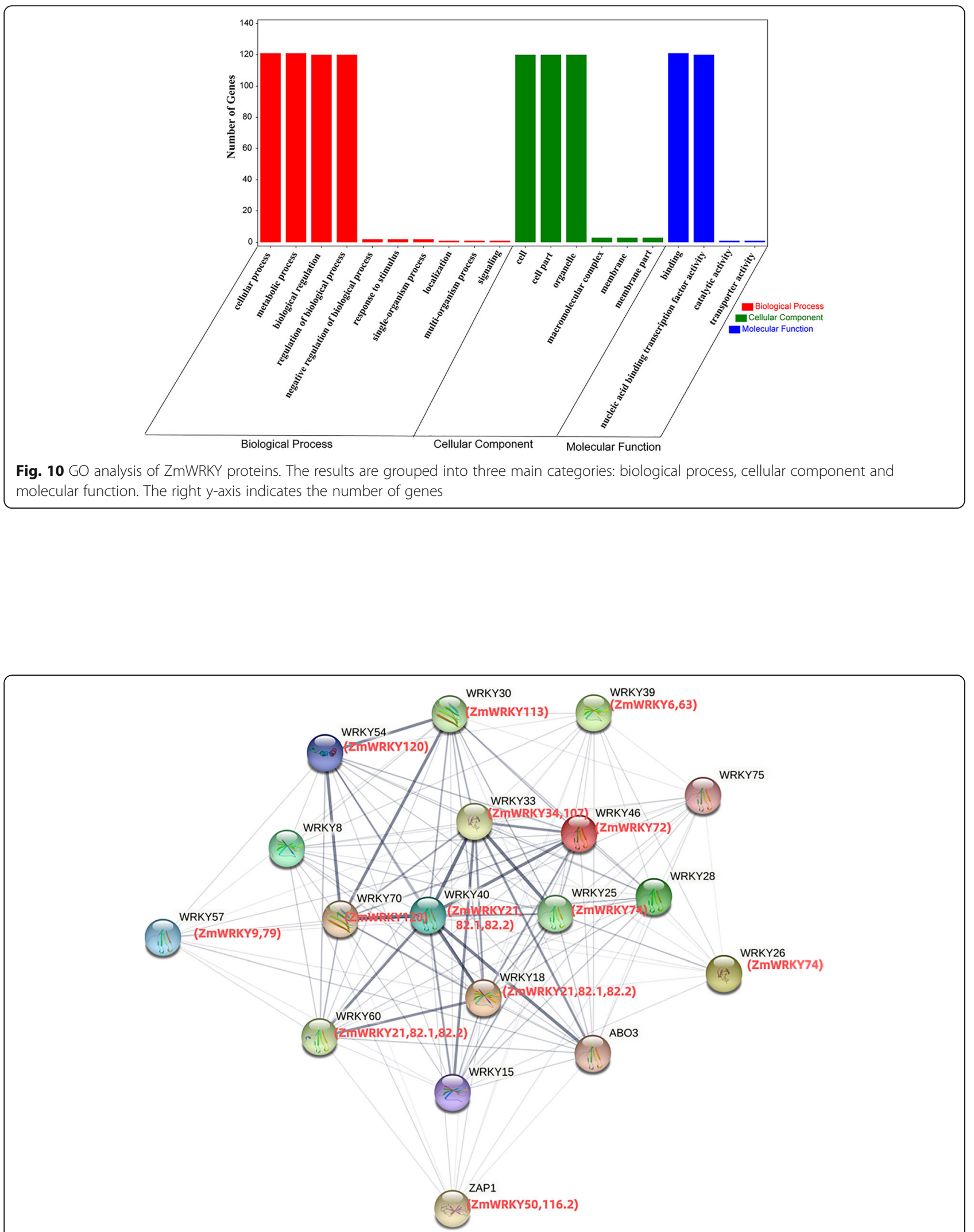

Fig. 11 Protein Protein Interaction network of specific ZmWRKY proteins. Black and red color characters represent Arabidopsis and maize, and the thick lines represent the high interaction 


\section{Discussion}

\section{Identification and characterization of ZmWRKY genes}

As far as is known, the WRKY gene family has been revealed to take part in regulating multiple biological processes especially in responses to various environmental stresses [26, 27]. The genome-wide analyses of WRKY gene families have been extensively performed in lots of higher plants whose whole genome sequencing have been completed, including Arabidopsis, rice, pear, grape and tomato [8, 23, 27-29]. In this study, a genome-wide analysis of maize WRKY genes was accomplished. A total of 125 WRKY genes were finally identified from the latest maize genome database. What's more, we also found that there were lots of alternative splice isoforms in $Z m W R K Y$ genes and their different transcripts were further analyzed including some properties listed in Table S1.

Multiple sequence alignment showed that most ZmWRKY proteins shared extremely conserved heptapeptides (WRKYGQK) at their N-terminals and a zincfinger-like structure $\left(\mathrm{C}_{2} \mathrm{H}_{2}\right.$ or $\mathrm{C}_{2} \mathrm{HC}$ type $)$ at their $\mathrm{C}$ terminals. Nonetheless, some variations, such as WKKY GQK, WRKYGEK and WRKYGKK, have also been revealed in this study. This phenomenon has also been discovered in the Arabidopsis, rice, grape, potato and apple WRKY members [8, 23, 28, 30, 31]. It is speculated that these variations have the possibility to change the binding specificity of DNA targets and influence the expression status of stress-responsive genes targeted by ZmWRKY TFs. In addition, it was revealed that seven ZmWRKY proteins that did not have a whole zinc finger motif and three proteins without the complete WRKY GQK sequence were also identified as the ZmWRKY family members. The gain and loss of domain might be one of the reasons for the expansion of WRKY gene family in maize.

In this study, an unrooted phylogenetic tree was established by multiple sequence alignment of conserved WRKY domains from these identified WRKY proteins in maize and some representatives selected from Arabidopsis and rice WRKY proteins. As depicted in Fig. 1, the ZmWRKY proteins from the Groups IIa and IIb were closer together, while the Group IId was closely related with the Group IIe. For the break of the Groups IIa and IIb and the break of the Groups IId and IIe were much later than the other Groups in the ancestor of terrestrial plants [10], it was supposed that the Groups IIa and IIb, and the Group IId and IIe should be merged into two new subfamily, IIa $+\mathrm{b}$ and IId $+\mathrm{e}$, respectively $[11,32]$.

Previous studies showed that the analysis of gene structure and conserved motifs can offer some crucial clues to analyze the evolutionary relationship in a gene family [33]. The characterization of $Z m W R K Y$ s about gene structure and conserved motifs showed that gene structure and motifs were highly conserved among the members of the same group. Most ZmWRKY genes contained two introns, which is commonplace in other plants, such as sesame (33/71), chickpea (39/69), pear (59/103) and cassava (42/85) [29, 34-36]. Overall, the position and phase of introns in the same group presented a good similarity. For example, the Group I genes contained three to five introns, while most of the Group III genes shared two introns. Moreover, we identified 20 conserved motifs (ranging from 15 to 50 amino acid residues in length) in total among 140 ZmWRKY TFs (Table S2). As shown in Fig. 4, the conserved motifs were distributed in a phylogenetic group-specific manner for the proteins in the same group, implying that the molecular structure in the same group may be quite conserved in the process of evolution (Table S2; Fig. 4).

\section{Gene duplication events of ZmWRKY genes}

Gene duplication, including tandem or segmental duplication, is a frequent occurrence during angiosperm evolution, which usually is regarded as a critical mechanism associated with the expansion and complexity of gene families [37]. In this study, there were 52 gene pairs in total identified to take part in segmental duplication events among the $125 \mathrm{ZmWRKY}$ genes, but without a single tandemly-duplicated gene pair. This result implied that segmental duplication events have played a crucial part in the expansion of maize WRKY gene family.

In addition, we also investigated the orthologous WRKY gene pairs among maize, Arabidopsis and rice. Arabidopsis and rice are the most significant eudicot and monocot model plant species and gene functions in Arabidopsis and rice mostly have been adequately interpreted. As shown in Fig. S2, 95 (76\%) ZmWRKY genes have one or two putative orthologs in rice; however, only six (4.8\%) ZmWRKY genes have orthologs in Arabidopsis. The result was in accordance with the present explanation of plant phylogeny. Furthermore, the synteny analysis result suggested that the majority of $Z m W R K Y$ orthologs might emerge after the divergence of monocots and dicots. Furthermore, we also found that the six $Z m W R K Y$ genes which being synteny with AtWRKYs also shared relative orthologs in rice, thus it was suspected that these genes in these species might antedate the divergence of monocots and eudicots.

The analysis of $K a$ and $K s$ substitutions in duplicated genes is a useful way to research the evolution of important genes [38]. The $K a / K s$ ratios of the 52 duplicated pairs revealed that these gene pairs seemed to have gone through strong purifying selection. Purifying selection usually selectively removes harmful alleles with the passage of time [39], suggesting that the WRKY gene family may play an essential role in the development and survival of the maize plant, which makes it necessary to protect and propagate its members. Maize genome has undergone twice whole genome duplication, and the 
previous one happened about 55-70 Mya before the divergence of Gramineae while the latter one after tetraploidization occurred about 4.8 Mya [40, 41]. The computational results of the duplication dates of the 52 paralogous pairs demonstrated that the divergence time ranged from 9.6653 to 153.9372 Mya (Table S4), suggesting that most of the segmental duplication events in the maize WRKY family did not happen until the divergence of the grasses later.

\section{Expression profiles of maize WRKY genes under abiotic stress treatment}

The WRKY TFs regulate a series of biological processes and take part in lots of abiotic stress responses. It has been demonstrated that the Groups III and IId WRKY genes play a key role in plant development, stress response and even evolution. Transient expression researches on the Group III WRKY genes of $A$. thaliana revealed that these genes are components of various plant stress signaling pathway, no matter in compatible, incompatible or non-host interactions, suggesting their functional segregation [42]. The Group III WRKY genes were considered as the most vital group about gene family evolution and seemingly have played an important role during the adaptation and evolution of plants [10]. Additionally, a great deal of studies demonstrated that the Group IId WRKY proteins are important regulators in multiple biological processes in plants. Eleven members of the Group IId OsWRKY in rice showed significant change in expression levels under different abiotic (salt, drought, and cold) and biotic stresses [43]. In banana, the Group IId WRKY genes were abundant that control ethylene (ET)-related maturing, while the expression of 17 out of 25 Group IId genes was differently affected by ethylene [44]. In this study, the expression of twenty-one $Z m W R K Y$ genes randomly chosen from the Groups III and IId members of WRKY gene family in maize were subjected to salt and drought stress treatments from two different tissues and profiled by means of quantitative real-time PCR. The results demonstrated that most of these genes were differentially up-regulated at different time points under salt and drought stress treatments, while there still were few $\operatorname{ZmWRKY}$ genes down-regulated in two different tissues after stress treatments, suggesting that the maize $Z m W R K Y$ genes might have a positive or negative response to abiotic stress treatment, but their response changed with the degree of stress. However, the underlying mechanism of this phenomenon still remains to be further elucidated. Increasing evidence suggests that WRKY TFs not only participate in plant growth and development, but also show complex regulatory mechanisms and networks involved in external abiotic stresses in a time-dependent manner. Notwithstanding this, there is still one possibility that a function of WRKY TFs may vary as a consequence of environmental stress and in the process of adaptation [45]. Noticeably, in this study all these detected genes exhibited differential expression patterns in two different tissues under salt and drought stress treatments. It can be speculated that the majority of $Z m W R K Y s$ may play different roles in different tissues. One possible explanation might be the tissue-specific regulation. Moreover, many studies have demonstrated that WRKY genes are involved in responses to abiotic stresses, such as cold, drought and salt etc. in a variety of plant species [11]. In addition, through promoter analysis, many similar abiotic stress response cis-elements were revealed in the promoter regions of WRKY genes in various species, implying that most WRKY genes in plants might be involved in the transcriptional control of multiple abiotic stress responses. In this study, our analysis also uncovered many cis-elements related to drought, low temperature, salt and multiple hormone-related response elements in the $Z m W R K Y$ promoters, implying that most $Z m W R K Y$ genes were involved in the transcriptional control of defense and abiotic stress responses. The response of $Z m W R K Y$ genes to abiotic stresses can provide valuable clues to reveal the potential role of WRKY genes in maize. Many studies have revealed that WRKY genes were induced and exhibited up-regulated or down-regulated expression levels by drought, salt and exogenous hormones in a time-dependent manner, indicating that WRKY transcription factors were involved in the response to abiotic stresses through stress-related transcriptional regulatory elements. In this study, most $Z m W R K Y$ genes can be induced by diverse stress treatments such as salt and drought, implying that a single WRKY gene can be regulated by various abiotic stress responses through different cis-elements in the promoter region. Previous studies have demonstrated that the analysis of ZmWRKY65 promoter sequence in maize indicated that it contains several stress-related transcriptional regulatory elements including ABA-responsive element, MYB-responsive element, MYCresponsive element, dehydration-responsive element and low temperature-responsive element recognition site sequences. Many environmental factors can induce the transcription of ZmWRKY65 gene, such as drought, salinity, high temperature and low temperature stresses. Moreover, the transcription of ZmWRKY65 gene was also affected by the induction of defense related plant hormones such as SA and exogenous ABA [46]. Furthermore, another two maize genes ZmWRKY40 and ZmWRKY106 were also identified to enhance the tolerances to drought and high-temperature $[47,48]$. The gene and promoter structure analyses showed that there was the conservative sequence and stress-related transcriptional regulatory elements between these genes. Taken together, the regulatory mechanism of WRKY genes in responding to different abiotic stresses is complicated, and the analysis of ZmWRKY gene expression profiles will 
offer new insights into figuring out signaling pathways in maize abiotic stress responses.

\section{Co-expression and protein protein interaction networks analysis}

Weighted Gene Co-Expression Network Analysis (WGCN

A) is usually used to analyze gene function and their connection from the overall biological function by identifying functionally related or similar gene components in highthroughput data. By using the method, researchers can discover the connections among the highly coordinated genes from different modules. The modules were defined as the clusters of highly interconnected genes, and the genes within the same cluster have high correlation coefficients among them. In this study, WGCNA was used to analyze gene expression profiles of maize WRKY genes from 60 diverse tissue samples in the database, and finally seven coexpression modules were constructed as shown in Fig. 9. In the black module, ZmWRKY21, ZmWRKY82.1, $Z m W R K Y 82.2$ and ZmWRKY83 had a certain relationship with each other. In addition, ZmWRKY21, ZmWRKY82.1 and ZmWRKY82.2 genes are orthologs of AtWRKY18, AtWRKY40 and AtWRKY60 genes in A. thaliana, respectively. Studies have shown that AtWRKY18, AtWRKY40 and AtWRKY60 worked in cluster, and were involved in the transcriptional regulation of ABFs/AREBs by binding to the W-box element present in the promoter [49]. The maize WRKY genes, which are similar to Arabidopsis orthologs, were revealed that they might have similar functions in the transcriptional regulation of ABFs/AREBs. The blue module (27 genes) was associated with the leaves (VT and R1), including ZmWRKY74 (the ortholog of AtWRKY25 and AtWRKY26) and ZmWRKY107 (the ortholog of AtWRKY33). It has been reported that the cross-regulation between AtWRKY25, AtWRKY26 and AtWRKY33 was necessary to promote the heat tolerance of plants [50]. Thus, it was speculated that along with them ZmWRKY74 and ZmWRKY107 might act as transcriptional regulators in maize response to heat stress. A growing study has demonstrated that the plant WRKY genes was involved in diverse biotic/abiotic stress responses as well as in developmental/ physiological processes [11]. In the brown module, it included ZmWRKY109.2 (the ortholog of AtWRKY53) and ZmWRKY9 (the ortholog of AtWRKY57). Studies have shown that AtWRKY53 and AtWRKY57 were involved in regulating leaf senescence [51, 52]. In the process of JAinduced leaf senescence, it has been demonstrated that AtWRKY57 played an important role in leaf senescence as the convergence point of JA signaling pathway and auxin crosstalk. Moreover, this module also included ZmWRKY15.1 (the ortholog of AtWRKY12), ZmWRKY15.2 (the ortholog of AtWRKY12) and ZmWRKY28 (the ortho$\log$ of AtWRKY13). Studies have shown that AtWRKY12 and AtWRKY13 were involved in regulating flowering time
[53]. Overall, these putative $Z m W R K Y$ genes, which might be correlated with transcriptional regulation of maize development, were identified in the present study by constructing a co-expression network to further infer the potential roles for these $Z m W R K Y$ genes and verify the function of these candidate genes associated with plant development in future.

Furthermore, based on the functional annotation and interaction analysis of WRKY proteins from other species, the possible regulatory effects of ZmWRKY proteins can be predicted. Five ZmWRKYs (ZmWRKY34, ZmWRKY107, ZmWRKY21, ZmWRKY82.1 and ZmWRKY82.2) with high sequence similarity with AtWRKY33 and AtWRKY40 were set as the central nodes of the interaction network. These five proteins were predicted to interact with other proteins according to varying degrees. The amino acid sequences of ZmWRKY21, ZmWRKY82.1 and ZmWRKY82.2 were similar to that of AtWRKY18/AtWRKY40/AtWRKY60, respectively. In Arabidopsis, AtWRKY18, AtWRKY40 and AtWRKY60 form homodimers or heterodimers to change the resistance to pathogens [54]. Furthermore, AtWRKY25 (ZmWRKY74), AtWRKY26 (ZmWRKY74) and AtWRKY33 (ZmWRKY107) actively regulate the synergy between ethylene activation and heat shock protein-related signaling pathways, which mediate the response to heat stress. These three proteins interact functionally and play a synergistic role in plant heat tolerance [50]. It is generally acknowledged that homologous proteins with similar domains and sequences among different species may share the same or similar functions. Overall, the results showed that diverse interactions among ZmWRKY proteins, which were similar to the results of co-expression network analysis, demonstrating the co-expression of ZmWRKY proteins in response to multiple biotic and abiotic stresses.

\section{Conclusions}

In conclusion, we identified 140 WRKY TFs altogether in maize. The classification, evolutionary characteristics, conserved domain and gene structure of the WRKY gene family in maize, together with stress-responsive ciselements in the promoters of $125 \mathrm{ZmWRKY}$ genes were investigated. The analyses of expression profiles based on RNA-seq method revealed their probable functions in different tissues. What's more, twenty-one ZmWRKY genes were activated by salt and drought stresses, implying their potential roles in abiotic stress responses of Zea mays $L$. Finally, via analyzing the expression patterns of $Z m W R K Y$ genes in diverse tissue types and construction of co-expression networks of ZmWRKY genes, subcellular localization prediction, GO annotation and PPI analysis of ZmWRKY proteins, a comprehensive overview has been provided for further exploring the function and regulatory mechanism of ZmWRKY genes in maize, which will help in elucidating their exact function in maize. 


\section{Materials and methods}

Plant materials, abiotic treatment, and tissue collection

The maize seeds in this experiment were provided from National Engineering Laboratory of Crop Stress Resistance Breeding, Anhui Agricultural University. Maize (Zea mays L. inbred line B73) plants were cultivated in an artificial climate chamber at $28{ }^{\circ} \mathrm{C}$ with long-day conditions of $14 \mathrm{~h}$ of light and $10 \mathrm{~h}$ of dark and an environmental humidity of $50 \%$. To impose the drought treatment, we gently pulled the whole maize seedlings out of the soil. For the salt-stress treatment, the maize seedling roots were submerged in a $200 \mathrm{mM} \mathrm{NaCl}$ solution. Seedlings maintained with enough water at $28^{\circ} \mathrm{C}$ in the dark were used as controls. The seedlings were collected at 1, 2, 4, and $8 \mathrm{~h}$ after the drought or salt treatment. Then the samples of seedling roots and leaves were rapidly frozen in liquid nitrogen, and immediately stored at $-80^{\circ} \mathrm{C}$ until RNA extraction.

\section{Identification of WRKY genes in maize}

The latest DNA and protein sequence information of B73 maize were obtained from phytozome 12.1 (http:// www.phytozome.net) [55]. A similar approach was used to identify the maize WRKY proteins just as reported in other plants [56]. The Hidden Markov Model (HMM) profile for the WRKY domain from the Pfam database (http://pfam.janelia.org) was used to identify WRKY proteins from the maize genome. Later, the Pfam database was used to check if all candidates contained the WRKY domain. In the end, the overlapping and deficient sequences were excluded through manual inspection in MEGA 7.0 [57]. The basic information of maize WRKY TFs such as the protein sequence length, open reading frame (ORF) length and chromosome location was got from the online project MaizeGDB (https://chinese.maizegdb.org). The molecular weight $(\mathrm{kDa})$ and isoelectric point $(\mathrm{PI})$ of each WRKY protein were estimated by the online ExPASy Bioinformatics Resource Portal (https://web.expasy.org/ compute_pi/) [58] and parameter was set as default.

\section{Sequence alignment and phylogenetic analysis}

The conserved WRKY domain sequences of ZmWRKY proteins were aligned by DNAMAN 7.0 software. Moreover, the conserved domains were investigated by online tool WebLogo (http://weblogo.berkeley.edu). The phylogenetic tree was constructed through the neighborjoining (NJ) method and a bootstrap value of 1000 by the MEGA 7.0 software [57]. To get a more reliable and credible result of classification of the different groups, 36 selected WRKY domains from rice and Arabidopsis were covered during phylogenetic analysis. Furthermore, an online program, Interactive Tree of Life (iTOL) (http://itol.embl.de/), was employed to give the phylogenetic tree a good looking.

\section{Chromosomal mapping, gene duplication and synteny analysis}

The chromosome mapping of $Z m W R K Y$ genes was accomplished using MG2C (http://mg2c.iask.in/mg2c_v2. $0 /$ ) based on their starting and ending positions in maize chromosomes. MCScanX was applied to identify tandem and segmental duplications of $Z m W R K Y$ genes [59] and the results of duplication genes were displayed by TBtools [60]. Each orthologous gene pair was then further investigated with PAL2NAL (http://www.bork.embl. $\mathrm{de} / \mathrm{pal} 2 \mathrm{nal} /$ ) [61] to calculate the Ks (synonymous substitution rate) and the Ka (non-synonymous substitution rate). The values of Ks were further used to calculate the divergence time $(\mathrm{T})$ by applying a formula $\mathrm{T}=\mathrm{Ks} / 2 \lambda \times$ $10^{-6}$ Mya, supposing a rate $(\lambda)$ of $6.5 \times 10^{-9}$ substitutions per synonymous site per year for maize [62]. MCScanX was also employed to carry out synteny analysis of ZmWRKY genes between Arabidopsis and rice.

Analysis of conserved motif distribution and gene structure The MEME Suite version 5.0.5 (http://meme.nbcr.net/ meme/) was applied to analyze conserved motifs for each ZmWRKY TF. The parameters for motif identification were set as the following: maximum number, 20; number of repetitions, any; the width of each motif, ranged from 6 to 100. By the use of the online program GSDS (http://gsds.cbi.pku.edu.cn/index.php) [33], gene structure diagrams were constructed utilizing the relevant nucleotide sequences of $Z m W R K Y$ genes gained from phytozome 12.1 (http://www.phytozome. net) [55].

\section{Analysis of the cis-regulatory elements in ZmWRKY genes} To survey the cis-acting factors of the promoter sequence, the online tool PlantCARE (http:// bioinformatics.psb.ugent.be/webtools/plantcare/html/)

[63] was applied to explore the genomic sequence in 2 $\mathrm{kb}$ upstream region of the initiation codon (ATG) in each $Z m W R K Y$ gene.

\section{Expression profile analysis of maize WRKY genes}

The standardized data (Reads/kb/Million, RPKM) for different tissues from different growth stages was provided by Sekhon et al. [64], and downloaded from MaizeGDB (http://www.maizegdb.org). Finally, we identified expression patterns of $Z m W R K Y$ genes in ten different tissues, including seed, root, seedling, stem, shoot tip, silks, leaf, tassel, husk and endosperm. The expression values were calculated by $\log 2$ (FPKM) and were displayed as a heat map by TBtools software [60].

\section{Analysis of maize WRKY gene expression under salt and drought stresses by qRT-PCR}

Total RNAs were extracted from different seedling samples using RNAiso Plus (TaKaRa, Japan) in accordance with the 
specification. Then the RNAs were reverse transcribed into the first-strand cDNA by FastKing RT Kit (With gDNase) (TIANGEN, China). To carry on the expression profile analysis of $Z m W R K Y$ genes under salt or drought stress treatment, several WRKY genes from group III and IId were selected for research by Real-time quantitative PCR (qPCR). Three replicates were conducted on a CFX96 Touch $^{\text {Tx }}$ RealTime PCR detection system (BIO-RAD, USA). The maize actin gene (Accession No.: NC_024466) was used as a reference for normalization. PCR reactions used $\mathrm{iTaq}^{\mathrm{Tm}}$ Universal SYBR $^{\circ}$ Green Supermix (BIO-RAD, USA). Amplification conditions were as follows: initial denaturation of $95^{\circ} \mathrm{C}$ for $30 \mathrm{~s}$ and 40 cycles of denaturation at $95^{\circ} \mathrm{C}$ for $5 \mathrm{~s}, 55^{\circ} \mathrm{C}$ annealing for $20 \mathrm{~s}$ and extending at $72^{\circ} \mathrm{C}$ for $30 \mathrm{~s}$, in the end, with a melting curve to check the amplification specificity. The relative mRNA expression level for each gene was calculated as $2^{-\Delta \Delta C T}$ method [65]. Gene-specific DNA primers for qPCR are presented in Supplementary Table 3. In the end, the mean $\Delta \mathrm{Ct}$ values were statistically analyzed using the Student's t-test (http://www.physics.csbsju.edu/stats/ttest _bulk_form.html) to identify the expression profiles showing significant differences. $P \leq 0.05$ was regarded as statistically significant.

\section{Weighted gene co-expression network construction}

The standardized data (Reads/kb/Million, RPKM) for different tissues from different growth stages were provided by Sekhon et al. [64], and downloaded from MaizeGDB (http://www.maizegdb.org). These data include 60 samples and more than 70,000 genes. The sample data of ZmWRKY genes were selected for subsequent analysis. R statistical software (version 4.0) and WGCNA package were used for statistical calculations [66]. By choosing an appropriate weighting coefficient $\beta$ (soft threshold), the connection between genes in the constructed network was subject to scale-free network distribution, and the correlation coefficient between genes was used to construct a hierarchical clustering tree. Different branches of the clustering tree were exhibited to represent different gene modules with different colors. Then, based on the weighted correlation coefficients of genes, the genes were classified according to their expression patterns, and the genes with similar patterns were grouped into one module, so that the genes were classified into different modules by gene expression patterns for further analysis. Finally, the coefficient was used to convert the similarity matrix into an adjacency matrix, and further into a topological overlap matrix (TOM), all genes were used as a TOM heap map to prove the high degree of independence between modules and the expression of genes in each module relative independence. Connectivity was defined as the sum of the weights across all the edges of a node, and the coexpression network was built using Cytoscape software.

\section{Subcellular localization of ZmWRKY proteins}

The online software WOLF PSORT (https://wolfpsort. hgc.jp/) was utilized to predict subcellular localization by uploading protein sequences.

\section{Gene ontology annotation and protein protein interaction analysis}

The GO number of the maize WRKY genes was obtained from Ensemble Plant (http://plants.ensembl.org/ index.html). The hierarchical structure file was loaded in Gene Ontology website (http://geneontology.org/). Furthermore, an online tool, OmicShare (https://www. omicshare.com/tools/), was used for plotting Gene Ontology annotation results. A functional protein association network was constructed in the STRING program based on the Arabidopsis association model with the confidence parameter for 0.15 and the number of interactions for 5 , respectively.

\section{Abbreviations}

ABA: Abscisic acid; qRT-PCR: Quantitative RT-PCR; WGCNA: Weighted gene coexpression network analysis; GO: Gene Ontology; PPI: Protein-protein interaction; ET: Ethylene; JA: Jasmonic acid; HMM: Hidden Markov Model; NJ: Neighbor-joining; Ks: synonymous substitution rate; Ka: non-synonymous substitution rate

\section{Supplementary Information}

The online version contains supplementary material available at https://doi. org/10.1186/s12870-021-03206-z.

\section{Additional file 1.}

\section{Data availability}

The datasets generated during the current study are available in the GenBank repository, http://www.ncbi.n/m.nih.gov/Genbank and the MaizeGDB, http://www.maizegdb.org and their public access to these databases are open. All data generated or analyzed during this study are included in this article and its supplementary information files. The accession numbers are as follows: Zm00001d028962, Zm00001d029564,

Zm00001d030139, Zm00001d030969, Zm00001d032265, Zm00001d033291, Zm00001d033471, Zm00001d033965, Zm00001d034073, Zm00001d034084, Zm00001d034475, Zm00001d034888, Zm00001d002405, Zm00001d002452, Zm00001d002794, Zm00001d002794, Zm00001d003331, Zm00001d004086, Zm00001d005056, Zm00001d005057, Zm00001d005622, Zm00001d005749, Zm00001d006001, Zm00001d006702, Zm00001d007329, Zm00001d039245, Zm00001d039531, Zm00001d039532, Zm00001d039584, Zm00001d040554, Zm00001d041397, Zm00001d041740, Zm00001d041958, GRMZM2G178671, Zm00001d043025, Zm00001d043060, Zm00001d043062, Zm00001d043063, Zm00001d043066, Zm00001d043474, Zm00001d043569, Zm00001d043663, Zm00001d043950, Zm00001d044010, Zm00001d044162, Zm00001d044171, Zm00001d044315, Zm00001d044682, Zm00001d044680, Zm00001d049173, Zm00001d050023, Zm00001d050195, Zm00001d050247, Zm00001d051328, Zm00001d051550, Zm00001d052357, Zm00001d052358, Zm00001d052355, Zm00001d005056, Zm00001d052847, Zm00001d053369, Zm00001d013307, Zm00001d013630, Zm00001d013709, Zm00001d015515, Zm00001d016052, Zm00001d016457, Zm00001d017712, Zm00001d035323, Zm00001d036726, Zm00001d037054, Zm00001d037607, Zm00001d037854, Zm00001d038023, Zm00001d038451, Zm00001d038761, Zm00001d038843, Zm00001d039032, Zm00001d039044, Zm00001d018656, Zm00001d020136, Zm00001d020137, Zm00001d020492, Zm00001d020495, Zm00001d020881, Zm00001d021947, Zm00001d022437, Zm00001d008190, Zm00001d008578, Zm00001d008793, Zm00001d008794, Zm00001d009595, Zm00001d009619, Zm00001d009698, Zm00001d009939, Zm00001d020137, Zm00001d010399, Zm00001d010616, Zm00001d010617, Zm00001d010805, Zm00001d011133, Zm00001d011237, 
Zm00001d011403, Zm00001d011413, Zm00001d011496, Zm00001d011527, GRMZM2G045560, Zm00001d012482, Zm00001d012505, Zm00001d012508, Zm00001d012507, Zm00001d012746, Zm00001d012789, Zm00001d045283, Zm00001d045375, Zm00001d046805, Zm00001d047309, Zm00001d047574, Zm00001d023332, Zm00001d023336, Zm00001d023615, Zm00001d024323, Zm00001d024376, Zm00001d025669, Zm00001d026218, Zm00001d026252.

\section{Authors' contributions}

These studies were designed by QY. HW, RQ, CY and XG carried out all the experimental analyses and prepared all figures and tables. The manuscript was drafted by HW and RQ. All authors have commented, read and approved the final manuscript.

\section{Funding}

This study was supported by grants from the National Natural Science Foundation of China (NSFC) (Grant NO.31571673) and the open fundings of National Engineering Laboratory of Crop Stress Resistance Breeding (Grant NO.KNZJ1023) and Anhui Provincial Key Laboratory of the Conservation and Exploitation of Biological Resources (Grant NO.Swzy202003) and Anhui Provincial Academic Funding Project for Top Talents in Disciplines (Majors) (Grant NO.gxbjZD 2021044). The funding agencies had no role in the design, analysis and interpretation of the data or writing of the manuscript.

\section{Declarations}

\section{Ethics approval and consent to participate}

This article does not contain any studies with human participants or animals performed by the authors. These methods were carried out in accordance with relevant guidelines and regulations including the IUCN Policy Statement on Research Involving Species at Risk of Extinction and the Convention on the Trade in Endangered Species of Wild Fauna and Flora. We confirm that all experimental protocols were approved by Anhui Agriculture University and Anhui Normal University.

\section{Consent for publication}

Not applicable.

\section{Competing interests}

The authors declare that they have no competing interests.

Received: 30 May 2021 Accepted: 2 September 2021

\section{Published online: 20 September 2021}

\section{References}

1. Cairns J, Prasanna BM. Developing and deploying climate-resilient maize varieties in the developing world. Curr Opin Plant Biol. 2018;45. https://doi. org/10.1016/j.pbi.2018.05.004.

2. Cairns J, Sonder K, P H Z, Verhulst N, Mahuku G, Babu R, Nair S, Das B, Govaerts B, Vinayan M et al. Maize Production in a Changing Climate: Impacts, Adaptation, and Mitigation Strategies. Advances in Agronomy. 2012; 114. https://doi.org/10.1016/B978-0-12-394275-3.00006-7.

3. Lobell D, Schlenker W, Costa-Roberts J. Climate trends and global crop production since 1980. Science. 2011;333(6042):616-20. https://doi.org/1 $0.1126 /$ science. 1204531.

4. Wang C, Yang Q, Wang W, Li Y, Guo Y, Zhang D, et al. A transposondirected epigenetic change in $\mathrm{ZmCCT}$ underlies quantitative resistance to Gibberella stalk rot in maize. New Phytol. 2017;215(4). https://doi.org/1 $0.1111 /$ nph. 14688 .

5. Ulker B, Somssich I, Ülker B, Somssich IE. WRKY transcription factors: from DNA binding towards biological function. Curr Opin plant biol 7: 491-498. Curr Opin Plant Biol. 2004;7(5):491-8. https://doi.org/10.1016/j.pbi.2004.07. 012.

6. Zhao H, Wang S, Chen S, Jiang J, Liu G. Phylogenetic and stress-responsive expression analysis of 20 WRKY genes in Populus simonii $\times$ Populus nigra. Gene. 2015; 565(1). https://doi.org/10.1016/j.gene.2015.04.002.

7. Zentgraf $\mathrm{U}$, Laun T, Miao Y. The complex regulation of WRKY53 during leaf senescence of Arabidopsis thaliana. Eur J Cell Biol. 2009;89(2-3):133-7. https://doi.org/10.1016/j.ejcb.2009.10.014.

8. Eulgem T, Rushton PJ, Robatzek S, Somssich IE. The WRKY superfamily of plant transcription factors. Trends Plant Sci. 2000;5(5):199-206. https://doi. org/10.1016/S1360-1385(00)01600-9.
9. Jiang Y, Duan Y, Jia Y, Ye S, Zhu J, Zhang F, et al. Genome-wide identification and characterization of the WRKY transcription factor family and analysis of their expression in response to biotic and abiotic stresses. Genome. 2015;52(1):77-88. https://doi.org/10.1093/jxb/eru381.

10. Zhang $Y$, Wang $L$. The WRKY transcription factor superfamily: its origin in eukaryotes and expansion in plants. BMC Evol Bio. 2005;5(1):1-12. https:// doi.org/10.1186/1471-2148-5-1.

11. Chen F, Hu Y, Vannozzi A, Wu K, Cai H, Qin Y, et al. The WRKY transcription factor family in model plants and crops. Crit Rev Plant Sci. 2018;36(5-6):31135. https://doi.org/10.1080/07352689.2018.1441103.

12. Yoo S, Kim S-H, Kim M-J, Ryu C-M, Kim Y, Cho B, et al. Involvement of the OsMKK4-OsMPK1 Cascade and its downstream transcription factor OsWRKY53 in the wounding response in Rice. Plant Pathol J. 2014;30(2): 168-77. https://doi.org/10.5423/PP.OA.10.2013.0106.

13. Juntawong P, Sirikhachornkit A, Pimjan R, Sonthirod C, Sangsrakru D, Yoocha T, Tangphatsornruang S, Srinives P. Elucidation of the molecular responses to waterlogging in Jatropha roots by transcriptome profiling. Plant Sci. 2014; 5. https://doi.org/10.3389/fpls.2014.00658

14. Diao W, Snyder J, Wang S, Liu J, Pan B, Guo G, et al. Genome-wide identification and expression analysis of WRKY gene family in Capsicum annuum L. Front Plant Sci. 2016;7. https://doi.org/10.3389/fpls.2016.00211.

15. Scarpeci TE, Zanor MI, Mueller-Roeber B, Valle EM. Overexpression of AtWRKY30 enhances abiotic stress tolerance during early growth stages in Arabidopsis thaliana. Plant Mol Biol. 2013;83(3):265-77. https://doi.org/10.1 007/s11103-013-0090-8.

16. Raineri J, Wang S, Peleg Z, Blumwald E, Chan RL. The rice transcription factor OsWRKY 47 is a positive regulator of the response to water deficit stress. Plant Mol Biol. 2015;88(4):401-13. https://doi.org/10.1007/s11103-015-0329-7.

17. Rengasamy R, Jiang S, Nadimuthu K, Nori VP, Srinivasan R. A comprehensive transcriptional profiling of the WRKY gene family in rice under various abiotic and phytohormone treatments. Plant Cell Physiol. 2008;49(6):865-79. https://doi.org/10.1093/pcp/pcn061.

18. Wu H, Ni Z, Yao Y, Guo G, Sun Q. Cloning and expression profiles of 15 genes encoding WRKY transcription factor in wheat (Triticum aestivem L.). Prog Nat Sci. 2008;18(6):697-705. https://doi.org/10.1016/j.pnsc.2007.12.006.

19. Cai R, Dai W, Zhang C, Wang Y, Wu M, Zhao Y, et al. The maize WRKY transcription factor ZmWRKY17 negatively regulates salt stress tolerance in transgenic Arabidopsis plants. Planta. 2017;246(6):1215-31. https://doi.org/ 10.1007/s00425-017-2766-9.

20. Li H, Gao Y, Xu H, Dai Y, Deng D, Chen J. ZmWRKY33, a WRKY maize transcription factor conferring enhanced salt stress tolerances in Arabidopsis. Plant Growth Regul. 2013;70(3):207-16. https://doi.org/10.1007/ s10725-013-9792-9.

21. Cai R, Zhao $Y$, Wang $Y$, Lin $Y$, Cheng B. Overexpression of a maize WRKY 58 gene enhances drought and salt tolerance in transgenic rice. Plant Cell Tissue Org Cult. 2015;1 19(3):565-77. https://doi.org/10.1007/s11240-014-0556-7.

22. Wei K, Chen J, Chen Y, Wu L, Xie D. Molecular phylogenetic and expression analysis of the complete WRKY transcription factor family in maize. DNA Res. 2012;19(2):153-64. https://doi.org/10.1093/dnares/dsr048.

23. Ross CA, Yue L, Shen QJ. The WRKY gene family in Rice (Oryza sativa). J Integr Plant Biol. 2007;49(6):827-42. https://doi.org/10.1111/j.1744-7909.2007. 00504.X.

24. Holub EB. The arms race is ancient history in Arabidopsis, the wildflower. Nat Rev Genet. 2001;2(7):516-27. https://doi.org/10.1038/35080508.

25. Nekrutenko A, Makova KD, Li W-H. The K(a)/K(S) ratio test for assessing the protein-coding potential of genomic regions: an empirical and simulation study. Genome Res. 2002;12(1):198-202. https://doi.org/10.1101/gr.200901.

26. Belamkar V, Weeks NT, Bharti AK, Farmer AD, Graham MA, Cannon SB. Comprehensive characterization and RNA-Seq profiling of the HD-zip transcription factor family in soybean (Glycine max) during dehydration and salt stress. BMC Genomics. 2014;15(1):950. https://doi.org/10.1186/1471-21 64-15-950.

27. Chen L, Yang Y, Liu C, Zheng Y, Xu M, Wu N, et al. Characterization of WRKY transcription factors in Solanum lycopersicum reveals collinearity and their expression patterns under cold treatment. Biochem Biophys Res Commun. 2015;464(3):962-8. https://doi.org/10.1016/j.bbrc.2015.07.085.

28. Guo C, Guo R, Xu X, Gao M, Li X, Song J, et al. Evolution and expression analysis of the grape (Vitis vinifera L.) WRKY gene family. J Exp Bot. 2014; 65(6):1513-28. https://doi.org/10.1093/jxb/eru007.

29. Huang $X, L i K, X u X$, Yao Z, Jin C, Zhang $S$. Genome-wide analysis of WRKY transcription factors in white pear (Pyrus bretschneideri) reveals evolution 
and patterns under drought stress. BMC Genomics. 2015;16(1):1104. https:// doi.org/10.1186/s12864-015-2233-6.

30. Meng D, Li Y, Bai Y, Li M, Cheng L. Genome-wide identification and characterization of WRKY transcriptional factor family in apple and analysis of their responses to waterlogging and drought stress. Plant Physiol Biochem. 2016; 103:71-83. https://doi.org/10.1016/j.plaphy.2016.02.006

31. Zhang C, Wang D, Yang C, Kong N, Shi Z, Zhao P, et al. Genome-wide identification of the potato WRKY transcription factor family. PLoS One. 2017;12(7):e0181573. https://doi.org/10.1371/journal.pone.0181573.

32. Rushton $\mathrm{P}$, Somssich I, Ringler $\mathrm{P}$, Shen Q. WRKY transcription factors. Trends plant Sci. Trends Plant Sci. 2010;15(5):247-58. https://doi.org/10.1016/j.tpla nts.2010.02.006.

33. Hu B, Jin J, Guo A, Zhang H, Luo J, Gao G. GSDS 2.0: an upgraded gene feature visualization server. Bioinformatics. 2015;31(8):1296-7. https://doi. org/10.1093/bioinformatics/btu817.

34. Li D, Liu P, Yu J, Wang L, Dossa K, Zhang Y, et al. Genome-wide analysis of WRKY gene family in the sesame genome and identification of the WRKY genes involved in responses to abiotic stresses. BMC Plant Biol. 2017;17(1): 152. https://doi.org/10.1186/s12870-017-1099-y.

35. Wagas M, Azhar MT, Rana IA, Azeem F, Ali MA, Nawaz MA, et al. Genomewide identification and expression analyses of WRKY transcription factor family members from chickpea (Cicer arietinum L.) reveal their role in abiotic stress-responses. Genes Gen. 2019;41(4):467-81. https://doi.org/10.1 007/s13258-018-00780-9.

36. Wei Y, Shi H, Xia Z, Tie W, Ding Z, Yan Y, Wang W, Hu W, Li K. GenomeWide Identification and Expression Analysis of the WRKY Gene Family in Cassava. Front Plant Sci. 2016; 7(25). https://doi.org/10.3389/fpls.2016.00025.

37. Liu H, Yang W, Liu D, Han Y, Zhang A, Li S. Ectopic expression of a grapevine transcription factor VWWRKY11 contributes to osmotic stress tolerance in Arabidopsis. Mol Biol Rep. 2010;38(1):417-27. https://doi.org/1 0.1007/s11033-010-0124-0.

38. Hanada K, Shiu S-H, Li W-H. The nonsynonymous/synonymous substitution rate ratio versus the radical/conservative replacement rate ratio in the evolution of mammalian genes. Mol Biol Evol. 2007;24(10):2235-41. https:// doi.org/10.1093/molbev/msm152.

39. Biswas S, Akey JM. Genomic insights into positive selection. Trends Genet. 2006;22(8):437-46. https://doi.org/10.1016/j.tig.2006.06.005.

40. Swigonová Z, Lai J, Ma J, Ramakrishna W, Llaca V, Bennetzen JL, et al. Close split of sorghum and maize genome progenitors. Genome Res. 2004; 14(10A):1916-23. https://doi.org/10.1101/gr.2332504.

41. Wang X, Shi X, Hao B, Song G, Luo J. Duplication and DNA segmental loss in the rice genome: implications for diploidization. The New Phytologist. 2005;165(3):937-46. https://doi.org/10.1111/j.1469-8137.2004.01293.x.

42. Kalde M, Barth M, Somssich I, Lippok B. Members of the Arabidopsis WRKY group III transcription factors are part of different plant defense signaling pathways. Mole Plant Microbe Inter. 2003;16(4):295-305. https://doi.org/10.1 094/MPMI.2003.16.4.295

43. Ramamoorthy $R$, Jiang S, Kumar N, Venkatesh P, Ramachandran S. A comprehensive transcriptional profiling of the WRKY gene family in Rice under various abiotic and Phytohormone treatments. Plant Cell Physiol. 2008;49(6):865-79. https://doi.org/10.1093/pcp/pcn061.

44. Goel R, Pandey A, Trivedi P, Asif M. Genome-wide analysis of the Musa WRKY gene family: evolution and differential expression during development and stress. Front Plant Sci. 2016;7. https://doi.org/10.3389/ fpls.2016.00299.

45. Yan JY, Li CX, Sun L, Ren JY, Li GX, Ding ZJ, et al. A WRKY transcription factor regulates Fe translocation under Fe deficiency in Arabidopsis. Plant Physiol. 2016;171(3):2017-27. https://doi.org/10.1104/pp.16.00252.

46. Huo T, Wang CT, Yu TF, Wang DM, Li M, Zhao D, et al. Overexpression of ZmWRKY65 transcription factor from maize confers stress resistances in transgenic Arabidopsis. Sci Rep. 2021;11:4024. https://doi.org/10.1038/s41 598-021-83440-5.

47. Wang CT, Ru JN, Liu YW, Li M, Zhao D, Yang JF, et al. Maize WRKY transcription factor ZmWRKY106 confers drought and heat tolerance in transgenic plants. Int J Mol Sci. 2018;19(10):3046.. https://doi.org/10.3390/ ijms19103046.

48. Wang CT, Ru JN, Liu YW, Yang JF, Li M, Xu ZS, et al. The maize WRKY transcription factor ZmWRKY40 confers drought resistance in transgenic Arabidopsis. Int J Mol Sci. 2018;19(9):2580. https://doi.org/10.3390/ijms19092580.
49. Antoni R, Rodriguez L, Gonzalez-Guzman M, Pizzio GA, Rodriguez PL. News on ABA transport, protein degradation, and ABFs/WRKYs in ABA signaling. Curr Opin Plant Biol. 2011;14(5):547-53. https://doi.org/10.1016/j.pbi.2011.06.004.

50. Li S, Fu Q, Chen L, Huang W, Yu D. Arabidopsis thaliana WRKY25, WRKY26, and WRKY33 coordinate induction of plant thermotolerance. Planta. 2011; 233(6):1237-52. https://doi.org/10.1007/s00425-011-1375-2.

51. Jiang Y. Liang., Yang S. Arabidopsis WRKY57 functions as a node of convergence for Jasmonic acid- and auxin-mediated signaling in Jasmonic acid-induced leaf senescence. Plant Cell. 2014;26(1):230-45. https://doi.org/1 0.1105/tpc.113.117838

52. Miao Y, Zentgraf U. A HECT E3 ubiquitin ligase negatively regulates Arabidopsis leaf senescence through degradation of the transcription factor WRKY53. Plant J. 2010;63(2):179-88. https://doi.org/10.1111/j.1365-313X.2010. 04233.x.

53. Li W, Wang H, Yu D. The Arabidopsis WRKY transcription factors WRKY12 and WRKY13 oppositely regulate flowering under short-day conditions. Mol Plant. 2016;9(11):1492-503. https://doi.org/10.1016/j.molp.2016.08.003.

54. Xu X, Chen C, Fan B, Chen Z. Physical and functional interactions between pathogen-induced Arabidopsis WRKY18, WRKY40, and WRKY60 transcription factors. Plant Cell. 2006;18(5):1310-26. https://doi.org/10.1105/tpc.105.03 7523.

55. Schnable P, Ware D, Fulton R, Stein J, Wei F, Pasternak S, et al. The B73 maize genome: complexity, diversity, and dynamics. Science. 2009; 326(5956):1112-5. https://doi.org/10.1126/science.1178534.

56. Wu K, Guo Z, Wang H, Li J. The WRKY family of transcription factors in rice and and Arabidopsis and their origins. DNA Res. 2005;12(1):9-26. https://doi. org/10.1093/dnares/12.1.9.

57. Hall BG. Building phylogenetic trees from molecular data with MEGA. Molecular Biology Evolution. 2013;30(5):1229-35. https://doi.org/10.1093/ molbev/mst012

58. Gasteiger E, Gattiker A, Hoogland C, Ivanyi I, Appel RD, Bairoch A. ExPASy: the proteomics server for in-depth protein knowledge and analysis. Nucleic Acids Res. 2003;31(13):3784-8. https://doi.org/10.1093/nar/gkg563.

59. Wang Y, Tang H, Debarry JD, Tan X, Li J, Wang X, Lee T-h, Jin H, Marler B, Guo H et al. MCScanX: a toolkit for detection and evolutionary analysis of gene synteny and collinearity. Nucleic Acids Res. 2012; 40(7):e49. https://doi. org/10.1093/nar/gkr1293

60. Chen C, Rui X, Hao C, He Y. TBtools, a toolkit for biologists integrating various HTS-data handling tools with a user-friendly interface. Mol Plant. 2020;13(8):1194-202. https://doi.org/10.1016/j.molp.2020.06.009.

61. Suyama M, Torrents D, Bork P. PAL2NAL: robust conversion of protein sequence alignments into the corresponding codon alignments. Nucleic Acids Res. 2006; 34(Web Server issue):W609-W612. https://doi.org/10.1093/ nar/gkl315.

62. Gaut BS, Morton BR, McCaig BC, Clegg MT. Substitution rate comparisons between grasses and palms: synonymous rate differences at the nuclear gene Adh parallel rate differences at the plastid gene rbcL. Proc Natl Acad Sci. 1996:93(19):10274-9. https://doi.org/10.1073/pnas.93.19.10274.

63. Lescot M. PlantCARE, a database of plant cis-acting regulatory elements and a portal to tools for in silico analysis of promoter sequences. Nucleic Acids Res. 2002;30(1):325-7. https://doi.org/10.1093/nar/30.1.325.

64. Sekhon RS, Lin H, Childs KL, Hansey CN, Kaeppler SM. Genome-wide atlas of transcription during maize development. Plant J. 2011;66(4):553-63. https:// doi.org/10.1111/j.1365-313X.2011.04527.x.

65. Livak KJ, Schmittgen TD. Analysis of relative gene expression data using real-time quantitative PCR and the $2-\Delta \Delta C T$ method. Methods. 2001;25(4): 402-8. https://doi.org/10.1006/meth.2001.1262.

66. Langfelder P, Horvath S. WGCNA: an R package for weighted correlation network analysis. BMC Bioinformatics. 2008;9(1):559. https://doi.org/10.11 86/1471-2105-9-559.

\section{Publisher's Note}

Springer Nature remains neutral with regard to jurisdictional claims in published maps and institutional affiliations. 